\title{
Infraparticles with Superselected Direction of Motion in Two-Dimensional Conformal Field Theory
}

\author{
Wojciech Dybalski ${ }^{1, \star}$, Yoh Tanimoto ${ }^{2, \star \star}$ \\ ${ }^{1}$ Zentrum Mathematik, Technische Universität München, 85747 Garching, Germany. \\ E-mail: dybalski@ma.tum.de \\ 2 Dipartimento di Matematica, Università di Roma “Tor Vergata”, Via della Ricerca Scientifica, \\ 00133 Roma, Italy. E-mail: tanimoto@mat.uniroma2.it
}

Received: 1 February 2011 / Accepted: 21 October 2011

Published online: 8 March 2012 - (C) The Author(s) 2012. This article is published with open access at Springerlink.com

\begin{abstract}
Particle aspects of two-dimensional conformal field theories are investigated, using methods from algebraic quantum field theory. The results include asymptotic completeness in terms of (counterparts of) Wigner particles in any vacuum representation and the existence of (counterparts of) infraparticles in any charged irreducible product representation of a given chiral conformal field theory. Moreover, an interesting interplay between the infraparticle's direction of motion and the superselection structure is demonstrated in a large class of examples. This phenomenon resembles the electron's momentum superselection expected in quantum electrodynamics.
\end{abstract}

\section{Introduction}

Particle aspects and superselection structure of quantum electrodynamics are plagued by the infrared problem, which has been a subject of study in mathematical physics for more than four decades [6-8, 10,16,18, 19,21-24,28-30,33,34,39-42,44,46,48]. The origin of this difficulty, inherited from classical electrodynamics, is the emission of photons which accompanies any change of the electron's momentum. It has two important consequences which are closely related: Firstly, the electron is not a particle in the sense of Wigner [50], but rather an infraparticle [46], i.e. it does not have a precise mass. Secondly, the electron's plane wave configurations of different momenta cannot be superposed into normalizable wavepackets. In fact, such configurations have different spacelike asymptotic flux of the electric field, which imposes a superselection rule [7]. The evidence for this phenomenon of the electron's momentum superselection comes from two sources: On the one hand, it appears in models of non-relativistic QED in the representation structure of the asymptotic electromagnetic field algebra [18]. On the other hand, it is suggested by structural results in the general framework of algebraic

\footnotetext{
* Supported by the DFG grant SP181/25.

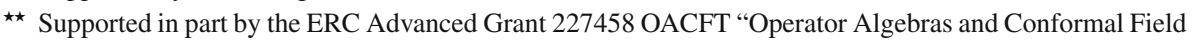
Theory".
} 
quantum field theory $[7,8,16,41,42]$. However, no examples of local, relativistic theories, describing infraparticles with superselected momentum, have been given to date. Thus the logical consistency of this property with the basic postulates of quantum field theory remains to be settled. As a step in this direction, we demonstrate in the present paper that a simple variant of this phenomenon - superselection of direction of motion - occurs in a large class of two-dimensional conformal field theories.

Conformal field theory has been a subject of intensive research over the last two decades, both from physical and mathematical viewpoints, motivated, in particular, by the search for non-trivial quantum field theories. (See e.g. [3] and references therein). It exhibits particularly interesting properties in two dimensions, where the symmetry group is infinite dimensional. Since the seminal work of Buchholz, Mack and Todorov [15] the superselection structure of these theories has been investigated [31] and deep classification results have been obtained [36,37]. It has remained unnoticed, however, that two-dimensional conformal field theories have also a rich and interesting particle structure: The concepts of Wigner particles and infraparticles have their natural counterparts in this setting and both types of excitations appear in abundance: Any chiral conformal field theory in a vacuum representation has a complete particle interpretation in terms of Wigner particles. Although such theories are non-interacting, their (Grosse-Lechner) deformations [14] exhibit non-trivial scattering and inherit the property of asymptotic completeness as we show in a companion paper [25]. It is verified in the present work that any charged irreducible product representation of a chiral conformal field theory admits infraparticles. In a large class of examples these infraparticles have superselected direction of motion, i.e. their plane wave configurations with opposite directions of momentum cannot be superposed. Thus subtle particle phenomena, which are not under control in physical spacetime, can be investigated in these two-dimensional models.

To keep our analysis general, we rely on the setting of algebraic QFT [32]. We base our discussion on the concept of a local net of $C^{*}$-algebras on $\mathbb{R}^{2}$, defined precisely in Subsect. 2.1: To any open, bounded region $\mathcal{O} \subset \mathbb{R}^{2}$ we attach a $C^{*}$-algebra $\mathfrak{A}(\mathcal{O})$, acting on a Hilbert space $\mathcal{H}$ of physical states. This algebra is generated by observables which can be measured with an experimental device localized in $\mathcal{O}$. It is contained in the quasilocal algebra $\mathfrak{A}$, which is the inductive limit of the net $\mathcal{O} \rightarrow \mathfrak{A}(\mathcal{O})$. Moreover, there acts a unitary representation of translations $\mathbb{R}^{2} \ni x \rightarrow U(x)$ on $\mathcal{H}$, whose adjoint action $\alpha_{x}(\cdot)=U(x) \cdot U(x)^{*}$ shifts the observables in spacetime. The infinitesimal generators of $U$ are interpreted as the Hamiltonian $H$ and the momentum operator $\boldsymbol{P}$. Their joint spectrum is contained in the closed forward lightcone $V_{+}$, to ensure the positivity of energy. If there exists a cyclic, unit vector $\Omega \in \mathcal{H}$ which is the unique (up to a phase) joint eigenvector of $H$ and $\boldsymbol{P}$ with eigenvalue zero, then we say that the theory is in a vacuum representation. If each of the subspaces $\mathcal{H}_{ \pm}=\operatorname{ker}(H \mp \boldsymbol{P})$ includes some vectors orthogonal to $\Omega$, then we say that the theory contains Wigner particles. Since we do not assume that these particles are described by vectors in some irreducible representation space of the Poincare group, the present definition is less restrictive than the conventional one. However, it is better suited for a description of the dispersionless kinematics of two-dimensional massless excitations. In particular, it allows us to apply the natural scattering theory, developed by Buchholz in [5], which we outline in Subsect. 2.2 below. We recall that in [5] these excitations are called 'waves', to stress their composite character.

Due to this compound structure of Wigner particles (or 'waves'), asymptotic completeness in a vacuum representation is not in conflict with the existence of charged representations with a non-trivial particle content. However, in charged representations of 
massless two-dimensional theories Wigner particles may be absent, as noticed in [12]. In this case scattering theory from [5] does not apply and an appropriate framework for the analysis of particle aspects is the theory of particle weights [10,16,23,24,35,41,42,47], developed by Buchholz, Porrmann and Stein, which we revisit in Subsects. 2.3 and 2.4. This theory is based on the concept of the asymptotic functional, given by

$$
\sigma_{\Psi}^{\text {out }}(C)=\lim _{t \rightarrow \infty} \int d x\left(\Psi \mid \alpha_{(t, x)}(C) \Psi\right)
$$

for any vector $\Psi \in \mathcal{H}$ of bounded energy, and suitable observables $C \in \mathfrak{A}$. (In general, some time averaging and restriction to a subnet may be needed before taking the limit). We remark for future reference that this functional induces a sesquilinear form $\psi_{\Psi}^{\text {out }}$ on a certain left ideal of $\mathfrak{A}$. We show in Theorem 2.11 below, that asymptotic functionals are non-zero in theories of Wigner particles. If non-trivial asymptotic functionals arise in the absence of Wigner particles, then we say that the theory describes infraparticles. ${ }^{1}$ Using standard decomposition theory, the GNS representation $\pi$ induced by the sesquilinear form $\psi_{\Psi}^{\text {out }}$ can be decomposed into a direct integral of irreducible representations

$$
\pi \simeq \int_{X} d \mu(\xi) \pi \xi,
$$

where $(X, d \mu)$ is a Borel space and $\simeq$ denotes unitary equivalence $[42,49]$. Results from $[2,47]$ suggest that the measurable field of irreducible representations $\left\{\pi_{\xi}\right\}_{\xi \in X}$ carries information about all the (infra-)particle types appearing in the theory. In particular, there exists a field of vectors $\left\{q_{\xi}\right\} \xi \in X$ which can be interpreted as the energy and momentum of plane wave configurations $\left\{\psi_{\xi}\right\}_{\xi \in X}$ of the respective (infra-)particles [42]. The sesquilinear forms $\left\{\psi_{\xi}\right\}_{\xi \in X}$, called pure particle weights, induce the representations $\left\{\pi_{\xi}\right\}_{\xi \in X}$ and satisfy

$$
\psi_{\Psi}^{\text {out }}=\int_{X} d \mu(\xi) \psi_{\xi}
$$

The existence of such a decomposition was shown, under certain technical restrictions, in $[41,42]$.

The theory of particle weights is sufficiently general to accommodate the phenomenon of the infraparticle's momentum superselection, discussed above: In this case $q_{\xi} \neq$ $q_{\xi^{\prime}}$ should imply that $\pi_{\xi}$ is not unitarily equivalent to $\pi_{\xi^{\prime}}$ for almost all labels $\xi$, $\xi^{\prime}$ corresponding to the infraparticle in question. Superselection of direction of motion is a milder property: It only requires that plane waves $\psi_{\xi}, \psi_{\xi^{\prime}}$, travelling in opposite directions, give rise to representations $\pi_{\xi}, \pi_{\xi^{\prime}}$ which are not unitarily equivalent. This latter interplay between the infraparticle's kinematics and the superselection structure occurs in some two-dimensional conformal field theories, as we explain below. We state this property precisely in Definitions 2.7 and 2.12, where we restrict attention to representations $\pi$ of (Murray-von Neumann) type I with atomic center. This is sufficient for our purposes and allows us to separate our central concept from ambiguities involved in the general decompositions (1.2), (1.3).

\footnotetext{
1 The conventional definition of infraparticles requires that both $\mathcal{H}_{+}$and $\mathcal{H}_{-}$contain at most multiples of the vacuum vector. Our (less restrictive) definition imposes this requirement on one of these subspaces only. Thus theories containing 'waves' running to the right but no 'waves' running to the left (or vice versa) describe infraparticles according to our terminology. Such nomenclature turns out to be more convenient in the context of two-dimensional, massless theories.
} 
Our discussion of conformal field theory relies on the notion of a local net of von Neumann algebras on $\mathbb{R}$ which we introduce in Subsect. 3.1. (Such nets arise e.g. by restricting the familiar Möbius covariant nets on the circle to the real line.) With any open bounded region $\mathcal{I} \subset \mathbb{R}$ we associate a von Neumann algebra $\mathcal{A}(\mathcal{I})$, acting on a Hilbert space $\mathcal{K}$, and denote the quasilocal algebra of this net by $\mathcal{A}$. Moreover, the Hilbert space $\mathcal{K}$ carries a unitary representation of translations $\mathbb{R} \ni s \rightarrow V(s)$, whose spectrum coincides with $\mathbb{R}_{+}$. If there exists a cyclic, unit vector $\Omega_{0} \in \mathcal{K}$, which is the unique (up to a phase) non-zero vector invariant under the action of $V$, then we say that the theory is in a vacuum representation. Given such a net, covariant under the action of some internal symmetry group, one can proceed to the fixed-point subnet which has a non-trivial superselection structure. In the simple case, considered in Subsect. 3.4, the action of $\mathbb{Z}_{2}$ is implemented by a unitary $W \neq I$ on $\mathcal{K}$ s.t. $W^{2}=I$. The fixed-point subnet $\mathcal{A}_{\mathrm{ev}}$ consists of all the elements of $\mathcal{A}$, which commute with $W$. The subspace $\mathcal{K}_{\mathrm{ev}}=\operatorname{ker}(W-I)\left(\right.$ resp. $\left.\mathcal{K}_{\text {odd }}=\operatorname{ker}(W+I)\right)$ is invariant under the action of $\mathcal{A}_{\mathrm{ev}}$ and gives rise to a vacuum representation (resp. a charged representation) of the fixed-point theory.

Given two nets of von Neumann algebras on the real line, $\mathcal{A}_{1}$ and $\mathcal{A}_{2}$, acting on Hilbert spaces $\mathcal{K}_{1}$ and $\mathcal{K}_{2}$, one obtains the two-dimensional chiral net $\mathfrak{A}$, acting on $\mathcal{H}=\mathcal{K}_{1} \otimes \mathcal{K}_{2}$, by the standard construction, recalled in Subsect. 3.1: The two real lines are identified with the lightlines in $\mathbb{R}^{2}$ and for any double cone $\mathcal{O}=\mathcal{I} \times \mathfrak{J}$ one sets ${ }^{2}$ $\mathfrak{A}(\mathcal{I} \times \mathfrak{J})=\mathcal{A}_{1}(\mathcal{I}) \otimes \mathcal{A}_{2}(\mathfrak{J})$. If the nets $\mathcal{A}_{1}, \mathcal{A}_{2}$ are in vacuum representations, with the vacuum vectors $\Omega_{1} \in \mathcal{K}_{1}, \Omega_{2} \in \mathcal{K}_{2}$, then $\mathfrak{A}$ is also in a vacuum representation, with the vacuum vector $\Omega=\Omega_{1} \otimes \Omega_{2}$. In spite of their simple tensor product structure, chiral nets play a prominent role in conformal field theory. In fact, with any local conformal net on $\mathbb{R}^{2}$ one can associate a chiral subnet by restricting the theory to the lightlines. In the important case of central charge $c<1$ these subnets were instrumental for the classification results, mentioned above, which clarified the superselection structure of a large class of models $[36,43]$. As we show in the present work, chiral nets also offer a promising starting point for the analysis of particle aspects of conformal field theories: Any chiral net in a vacuum representation is an asymptotically complete theory of Wigner particles. Moreover, any charged irreducible product representation of such a net contains infraparticles. With this information at hand, we exhibit examples of infraparticles with superselected direction of motion. This construction is summarized briefly in the remaining part of this Introduction.

Let us consider two fixed-point nets $\mathcal{A}_{1, \text { ev }}, \mathcal{A}_{2, \text { ev }}$, obtained from $\mathcal{A}_{1}$ and $\mathcal{A}_{2}$ with the help of the unitaries $W_{1}$ and $W_{2}$, implementing the respective actions of $\mathbb{Z}_{2}$. The resulting chiral net $\mathfrak{A}_{\mathrm{ev}}$ acts on the Hilbert space $\mathcal{H}=\mathcal{K}_{1} \otimes \mathcal{K}_{2}$, which decomposes into four invariant subspaces with different particle structure:

$$
\mathcal{H}=\left(\mathcal{K}_{1, \text { ev }} \otimes \mathcal{K}_{2, \text { ev }}\right) \oplus\left(\mathcal{K}_{1, \text { odd }} \otimes \mathcal{K}_{2, \text { ev }}\right) \oplus\left(\mathcal{K}_{1, \text { ev }} \otimes \mathcal{K}_{2, \text { odd }}\right) \oplus\left(\mathcal{K}_{1, \text { odd }} \otimes \mathcal{K}_{2, \text { odd }}\right)
$$

$\mathfrak{A}_{\mathrm{ev}}$ restricted to $\mathcal{H}_{0}:=\mathcal{K}_{1, \mathrm{ev}} \otimes \mathcal{K}_{2 \text {,ev }}$ is a chiral theory in a vacuum representation. Thus it is an asymptotically complete theory of Wigner particles, by the result mentioned above. $\mathcal{H}_{\mathrm{R}}:=\mathcal{K}_{1 \text {,odd }} \otimes \mathcal{K}_{2 \text {,ev }}$ contains 'waves' travelling to the right, but no 'waves' travelling to the left. In $\mathcal{H}_{\mathrm{L}}:=\mathcal{K}_{1 \text {,ev }} \otimes \mathcal{K}_{2 \text {,odd }}$ the opposite situation occurs. Thus $\mathfrak{A}_{\mathrm{ev}}$ restricted to $\mathcal{H}_{\mathrm{R}}$ or $\mathcal{H}_{\mathrm{L}}$ describes infraparticles, according to our terminology. Finally,

\footnotetext{
${ }^{2}$ In the main part of the paper $\mathfrak{A}(\mathcal{I} \times \mathfrak{J})$ denotes a suitable weakly dense 'regular subalgebra' of $\mathcal{A}_{1}(\mathcal{I}) \otimes$ $\mathcal{A}_{2}(\mathfrak{J})$. This distinction is not essential for the present introductory discussion.
} 
$\mathfrak{A}_{\mathrm{ev}}$ restricted to $\hat{\mathcal{H}}:=\mathcal{K}_{1, \text { odd }} \otimes \mathcal{K}_{2 \text {,odd }}$ is a theory of infraparticles which does not contain 'waves'. In Theorem 3.10 below, which is our main result, we establish superselection of direction of motion for infraparticles described by the net $\hat{\mathfrak{A}}=\left.\mathfrak{A}_{\mathrm{ev}}\right|_{\hat{\mathcal{H}}}$. The argument proceeds as follows: $\mathfrak{A}_{\mathrm{ev}}$ is contained in $\mathfrak{A}$, which is an asymptotically complete theory of Wigner particles. Thus we can use the scattering theory from [5] to compute the asymptotic functionals (1.1) and obtain the decompositions (1.2) of their GNS representations. Interpreted as a state on $\mathfrak{A}$, any vector $\Psi_{1} \otimes \Psi_{2} \in \hat{\mathcal{H}}$ consists of two 'waves' at asymptotic times: $\Psi_{1} \otimes \Omega_{2}$ travelling to the right and $\Omega_{1} \otimes \Psi_{2}$ travelling to the left. (cf. Theorem 3.3 below). However, these two vectors belong to different invariant subspaces of $\mathfrak{A}_{\mathrm{ev}}$, namely to $\mathcal{H}_{\mathrm{R}}$ and $\mathcal{H}_{\mathrm{L}}$. The corresponding representations of $\hat{\mathfrak{A}}$ are not unitarily equivalent, since they have different structure of the energy-momentum spectrum.

Our paper is organized as follows: Sect. 2, which does not rely on conformal symmetry, concerns two-dimensional, massless quantum field theories and their particle aspects: Preliminary Subsect. 2.1 introduces the main concepts. In Subsect. 2.2 we recall the scattering theory of two-dimensional, massless Wigner particles developed in [5]. Subsection 2.3 gives a brief exposition of the theory of particle weights and introduces our main concept: superselection of direction of motion. Subsect. 2.4 presents our main technical result, stated in Theorem 2.11, which clarifies the structure of asymptotic functionals in theories of Wigner particles. Its proof is given in Appendix A. In Sect. 3 we apply the concepts and tools presented in Sect. 2 to chiral conformal field theories. Our setting, which is slightly more general than the usual framework of conformal field theory, is presented in Subsect. 3.1. In Subsect. 3.2 we show that any chiral theory in a vacuum representation has a complete particle interpretation in terms of Wigner particles. In Subsect. 3.3 we demonstrate that charged irreducible product representations of any chiral theory describe infraparticles. Subsect. 3.4 presents our main result, that is superselection of the infraparticle's direction of motion in chiral theories arising from fixed-point nets of $\mathbb{Z}_{2}$ actions. Proofs of some auxiliary lemmas are postponed to Appendix B. In Sect. 4 we summarize our work and discuss future directions.

\section{Particle Aspects of Two-Dimensional Massless Theories}

2.1. Preliminaries. In this section, which does not rely on conformal symmetry, we present some general results on particle aspects of massless quantum field theories in two-dimensional spacetime. We rely on the following variant of the Haag-Kastler axioms [32]:

Definition 2.1. A local net of $C^{*}$-algebras on $\mathbb{R}^{2}$ is a pair ( $\left.\mathfrak{A}, U\right)$ consisting of a map $\mathcal{O} \rightarrow \mathfrak{A}(\mathcal{O})$ from the family of open, bounded regions of $\mathbb{R}^{2}$ to the family of $C^{*}$-algebras on a Hilbert space $\mathcal{H}$, and a strongly continuous unitary representation of translations $\mathbb{R}^{2} \ni x \rightarrow U(x)$ acting on $\mathcal{H}$, which are subject to the following conditions:

1. (isotony) If $\mathcal{O}_{1} \subset \mathcal{O}_{2}$, then $\mathfrak{A}\left(\mathcal{O}_{1}\right) \subset \mathfrak{A}\left(\mathcal{O}_{2}\right)$.

2. (locality) If $\mathcal{O}_{1} \perp \mathcal{O}_{2}$, then $\left[\mathfrak{A}\left(\mathcal{O}_{1}\right), \mathfrak{A}\left(\mathcal{O}_{2}\right)\right]=0$, where $\perp$ denotes spacelike separation.

3. (covariance) $U(x) \mathfrak{A}(\mathcal{O}) U(x)^{*}=\mathfrak{A}(\mathcal{O}+x)$ for any $x \in \mathbb{R}^{2}$.

4. (positivity of energy) The spectrum of $U$ is contained in the closed forward lightcone $V_{+}:=\left\{(\omega, \boldsymbol{p}) \in \mathbb{R}^{2}|\omega \geq| \boldsymbol{p} \mid\right\}$.

5. (regularity) The group of translation automorphisms $\alpha_{x}(\cdot)=U(x) \cdot U(x)^{*}$ satisfies $\lim _{x \rightarrow 0}\left\|\alpha_{x}(A)-A\right\|=0$ for any $A \in \mathfrak{A}$.

We also introduce the quasilocal $C^{*}$-algebra of this net $\mathfrak{A}=\overline{\bigcup_{\mathcal{O} \subset \mathbb{R}^{2}} \mathfrak{A}(\mathcal{O})}$. 
For any given net $(\mathfrak{A}, U)$ there exists exactly one unitary representation of translations $U^{\text {can }}$ s.t. $U^{\text {can }}$ implements $\alpha$, all the operators $U^{\text {can }}(x), x \in \mathbb{R}^{2}$ are contained in $\mathfrak{A}^{\prime \prime}$, the spectrum of $U^{\text {can }}$ is contained in $V_{+}$and has Lorentz invariant lower boundary upon restriction to any subspace of $\mathcal{H}$ invariant under the action of $\mathfrak{A}^{\prime \prime}$ [4]. We assume that this canonical representation of translations has been selected above, i.e. $U=U^{\text {can }}$. We denote by $(H, P)$ the corresponding energy-momentum operators, i.e. $U(x)=e^{i H t-i \boldsymbol{P x}}, x=(t, \boldsymbol{x})$. As we are interested in scattering of massless particles, we introduce the single-particle subspaces $\mathcal{H}_{ \pm}:=\operatorname{ker}(H \mp \boldsymbol{P})$ and denote the corresponding projections by $P_{ \pm}$. The intersection $\mathcal{H}_{+} \cap \mathcal{H}_{-}$contains only translationally invariant vectors. If $\mathcal{H}_{+} \neq \mathcal{H}_{+} \cap \mathcal{H}_{-}$and $\mathcal{H}_{-} \neq \mathcal{H}_{+} \cap \mathcal{H}_{-}$then we say that the theory describes Wigner particles. If $U$ has a unique (up to a phase) invariant unit vector $\Omega \in \mathcal{H}$ and $\Omega$ is cyclic under the action of $\mathfrak{A}$ then we say that the net $(\mathfrak{A}, U$ ) is in a vacuum representation. In this case $\mathfrak{A}$ acts irreducibly on $\mathcal{H}$ (cf. Theorem 4.6. of [1]). Scattering theory for Wigner particles in a vacuum representation, developed in [5], will be recalled in Subsect. 2.2.

In the absence of Wigner particles we will apply the theory of particle weights $[10,16,41,42]$, outlined in Subsect. 2.3, to extract the (infra-)particle content of a given theory. In this context it is necessary to consider various representations of the net $(\mathfrak{A}, U)$. A representation of the net $(\mathfrak{A}, U)$ is, by definition, a family of representations $\left\{\pi_{\mathcal{O}}\right\}$ of local algebras which are consistent in the sense that if $\mathcal{O}_{1} \subset \mathcal{O}_{2}$ then it holds that $\left.\pi_{\mathcal{O}_{2}}\right|_{\mathfrak{A}\left(\mathcal{O}_{1}\right)}=\pi_{\mathcal{O}_{1}}$. Since the family of open bounded regions in $\mathbb{R}^{2}$ is directed, this representation uniquely extends to a representation $\pi$ of the quasilocal $C^{*}$-algebra $\mathfrak{A}$. Conversely, a representation of $\mathfrak{A}$ induces a consistent family of representations of local algebras. In the following $\pi$ may refer to a representation of $\mathfrak{A}$ or a family of representations. We say that a representation $\pi: \mathfrak{A} \rightarrow B\left(\mathcal{H}_{\pi}\right)$ is covariant, if there exists a strongly continuous group of unitaries $U_{\pi}$ on $\mathcal{H}_{\pi}$, s.t.

$$
\pi\left(\alpha_{x}(A)\right)=U_{\pi}(x) \pi(A) U_{\pi}(x)^{*}, \quad A \in \mathfrak{A}, x \in \mathbb{R}^{2} .
$$

Moreover, we say that this representation has positive energy, if the joint spectrum of the generators of $U_{\pi}$ is contained in $V_{+}+q$ for some $q \in \mathbb{R}^{2}$. We denote the corresponding canonical representation of translations by $U_{\pi}^{\text {can }}$ and note that $\left(\pi(\mathfrak{A}), U_{\pi}^{\text {can }}\right)$ is again a local net of $C^{*}$-algebras in the sense of Definition 2.1. We say that the net $\left(\pi(\mathfrak{A}), U_{\pi}^{\text {can }}\right)$ is in a charged irreducible representation, if $\pi(\mathfrak{A})$ acts irreducibly on a non-trivial Hilbert space $\mathcal{H}_{\pi}$ which does not contain non-zero invariant vectors of $U_{\pi}^{\text {can }}$.

We call two representations $\left(\pi_{1}, \mathcal{H}_{\pi_{1}}\right)$ and $\left(\pi_{2}, \mathcal{H}_{\pi_{2}}\right)$ of $(\mathfrak{A}, U)$ unitarily equivalent, (in short $\left(\pi_{1}, \mathcal{H}_{\pi_{1}}\right) \simeq\left(\pi_{2}, \mathcal{H}_{\pi_{2}}\right)$ ), if there exists a unitary $W: \mathcal{H}_{\pi_{1}} \rightarrow \mathcal{H}_{\pi_{2}}$ s.t.

$$
W \pi_{1}(A)=\pi_{2}(A) W, \quad A \in \mathfrak{A} .
$$

If $\pi_{1}$ is a covariant, positive energy representation then so is $\pi_{2}$ and it is easy to see that

$$
W U_{\pi_{1}}^{\text {can }}(x)=U_{\pi_{2}}^{\text {can }}(x) W, \quad x \in \mathbb{R}^{2} .
$$

Remark 2.2. We note that our (non-standard) Definition 2.1 of the local net neither imposes the Poincaré covariance nor the existence of the vacuum vector. Thus it applies both to vacuum representations and charged representations, which facilitates our discussion. Apart from the physically motivated assumptions, we adopt the regularity property 5, which can always be assured at the cost of proceeding to a weakly dense subnet. This property seems indispensable in the general theory of particle weights [41], e.g. 
in the proof of Proposition 2.10 stated below. For consistency of the presentation, we proceed to regular subnets also in our discussion of conformal field theories in Sect. 3. We stress, however, that this property is not needed there at the technical level.

2.2. Scattering states. Scattering theory for Wigner particles in a vacuum representation of a two-dimensional massless theory $(\mathfrak{A}, U)$ was developed in [5]. For the reader's convenience we recall here the main steps of this construction. Following [5], for any $F \in \mathfrak{A}$ and $T \geq 1$ we introduce the approximants:

$$
F_{ \pm}\left(h_{T}\right)=\int h_{T}(t) F(t, \pm t) d t,
$$

where $F(x):=\alpha_{x}(F), h_{T}(t)=|T|^{-\varepsilon} h\left(|T|^{-\varepsilon}(t-T)\right), 0<\varepsilon<1$ and $h \in C_{0}^{\infty}(\mathbb{R})$ is a non-negative function s.t. $\int d t h(t)=1$. By applying the mean ergodic theorem, one obtains

$$
\lim _{T \rightarrow \infty} F_{ \pm}\left(h_{T}\right) \Omega=P_{ \pm} F \Omega .
$$

Moreover, for $F \in \mathfrak{A}(\mathcal{O})$ and sufficiently large $T$ the operator $F_{+}\left(h_{T}\right)\left(\right.$ resp. $F_{-}\left(h_{T}\right)$ ) commutes with any observable localized in the left (resp. right) component of the spacelike complement of $\mathcal{O}$. Exploiting these two facts, the following result was established in [5]:

Proposition 2.3 ([5]). Let $F, G \in \mathfrak{A}$. Then the limits

$$
\Phi_{ \pm}^{\text {out }}(F):=\underset{T \rightarrow \infty}{\mathrm{s}-\lim _{ \pm}} F_{ \pm}\left(h_{T}\right)
$$

exist and are called the (outgoing) asymptotic fields. They depend only on the respective vectors $\Phi_{ \pm}^{\text {out }}(F) \Omega=P_{ \pm} F \Omega$ and satisfy:

(a) $\Phi_{+}^{\text {out }}(F) \mathcal{H}_{+} \subset \mathcal{H}_{+}, \quad \Phi_{-}^{\text {out }}(G) \mathcal{H}_{-} \subset \mathcal{H}_{-}$.

(b) $\quad \alpha_{x}\left(\Phi_{+}^{\text {out }}(F)\right)=\Phi_{+}^{\text {out }}\left(\alpha_{x}(F)\right), \quad \alpha_{x}\left(\Phi_{-}^{\text {out }}(G)\right)=\Phi_{-}^{\text {out }}\left(\alpha_{x}(G)\right)$ for $x \in \mathbb{R}^{2}$.

(c) $\left[\Phi_{+}^{\text {out }}(F), \Phi_{-}^{\text {out }}(G)\right]=0$.

The incoming asymptotic fields $\Phi_{ \pm}^{\mathrm{in}}(F)$ are constructed analogously, by taking the limit $T \rightarrow-\infty$.

With the help of the asymptotic fields one defines the scattering states as follows: Since $\mathfrak{A}$ acts irreducibly on $\mathcal{H}$, for any $\Psi_{ \pm} \in \mathcal{H}_{ \pm}$we can find $F_{ \pm} \in \mathfrak{A}$ s.t. $\Psi_{ \pm}=F_{ \pm} \Omega$ [45]. The vectors

$$
\Psi_{+} \stackrel{\text { out }}{\times} \Psi_{-}=\Phi_{+}^{\text {out }}\left(F_{+}\right) \Phi_{-}^{\text {out }}\left(F_{-}\right) \Omega
$$

are called the (outgoing) scattering states. By Proposition 2.3 they do not depend on the choice of $F_{ \pm}$within the above restrictions. The incoming scattering states $\Psi_{+} \times \Psi_{-}$are defined analogously. The physical interpretation of these vectors, as two independent excitations travelling in opposite directions at asymptotic times, relies on the following proposition from [5]: 
Proposition 2.4 ([5]). Let $\Psi_{ \pm}, \Psi_{ \pm}^{\prime} \in \mathcal{H}_{ \pm}$. Then:

(a) $\left(\Psi_{+} \stackrel{\text { out }}{\times} \Psi_{-} \mid \Psi_{+}^{\prime} \stackrel{\text { out }}{\times} \Psi_{-}^{\prime}\right)=\left(\Psi_{+} \mid \Psi_{+}^{\prime}\right)\left(\Psi_{-} \mid \Psi_{-}^{\prime}\right)$,

(b) $U(x)\left(\Psi_{+} \stackrel{\text { out }}{\times} \Psi_{-}\right)=\left(U(x) \Psi_{+}\right) \stackrel{\text { out }}{\times}\left(U(x) \Psi_{-}\right)$, for $x \in \mathbb{R}^{2}$.

Analogous relations hold for the incoming scattering states.

Following [5], we define the subspaces spanned by the respective scattering states:

$$
\mathcal{H}^{\text {in }}=\mathcal{H}_{+} \times \mathcal{H}_{-} \quad \text { and } \mathcal{H}^{\text {out }}=\mathcal{H}_{+} \stackrel{\text { out }}{\times} \mathcal{H}_{-} .
$$

Next, we introduce the wave operators $\Omega^{\text {out }}: \mathcal{H}_{+} \otimes \mathcal{H}_{-} \rightarrow \mathcal{H}^{\text {out }}$ and $\Omega^{\text {in }}: \mathcal{H}_{+} \otimes \mathcal{H}_{-} \rightarrow$ $\mathcal{H}^{\text {in }}$, extending by linearity the relations

$$
\Omega^{\text {out }}\left(\Psi_{+} \otimes \Psi_{-}\right)=\Psi_{+} \stackrel{\text { out }}{\times} \Psi_{-} \text {and } \Omega^{\text {in }}\left(\Psi_{+} \otimes \Psi_{-}\right)=\Psi_{+} \times \Psi_{-}^{\text {in }} .
$$

These operators are isometric in view of Proposition 2.4 (a). The scattering operator $S: \mathcal{H}^{\text {out }} \rightarrow \mathcal{H}^{\text {in }}$, given by

$$
S=\Omega^{\text {in }}\left(\Omega^{\text {out }}\right)^{*},
$$

is also an isometry. Now we are ready to introduce two important concepts:

Definition 2.5. (a) If $S=I$ on $\mathcal{H}^{\text {out }}$, then we say that the theory is non-interacting.

(b) If $\mathcal{H}^{\text {in }}=\mathcal{H}^{\text {out }}=\mathcal{H}$ then we say that the theory is asymptotically complete (in terms of 'waves').

We show in Theorem 3.3 below that any chiral conformal field theory in a vacuum representation is both non-interacting and asymptotically complete. (We demonstrated these facts already in [25] in a different context.)

To conclude this subsection, we introduce some other useful concepts which are needed in Theorem 2.11 below: Let us choose some closed subspaces $\mathcal{K}_{ \pm} \subset \mathcal{H}_{ \pm}$, invariant under the action of $U$, and denote by $\mathcal{K}_{+} \stackrel{\text { out }}{\times} \mathcal{K}_{-}$the linear span of the respective scattering states. For any $\Psi \in \mathcal{K}_{+} \stackrel{\text { out }}{\times} \mathcal{K}_{-} \subset \mathcal{H}^{\text {out }}$ we introduce the positive functionals $\rho_{ \pm, \Psi}$, given by the relations

$$
\begin{aligned}
& \rho_{+, \Psi}(A)=\left(\left(\Omega^{\text {out }}\right)^{-1} \Psi \mid(A \otimes I)\left(\Omega^{\text {out }}\right)^{-1} \Psi\right), \\
& \rho_{-, \Psi}(A)=\left(\left(\Omega^{\text {out }}\right)^{-1} \Psi \mid(I \otimes A)\left(\Omega^{\text {out }}\right)^{-1} \Psi\right),
\end{aligned}
$$

where $A \in B(\mathcal{H})$ and the embedding $\mathcal{K}_{+} \otimes \mathcal{K}_{-} \subset \mathcal{H} \otimes \mathcal{H}$ is understood. These functionals can be expressed as follows

$$
\rho_{ \pm, \Psi}(\cdot)=\sum_{n \in \mathbb{N}}\left(\Psi_{ \pm, n} \mid \cdot \Psi_{ \pm, n}\right),
$$

where $\Psi_{ \pm, n} \in \mathcal{K}_{ \pm}$and $\sum_{n \in \mathbb{N}}\left\|\Psi_{ \pm, n}\right\|^{2}=\|\Psi\|^{2}$. It follows easily from Lemma A.2, that for $\Psi \in P_{E}\left(\mathcal{K}_{+} \stackrel{\text { out }}{\times} \mathcal{K}_{-}\right)$, where $P_{E}$ is the spectral projection on vectors of energy not larger than $E$, one can choose $\Psi_{ \pm, n} \in P_{E} \mathcal{K}_{ \pm}$. We note that for $\|\Psi\|=1$ the functionals $\rho_{ \pm, \Psi}$ are just the familiar reduced density matrices. 
2.3. Particle weights. Similarly as in the previous subsection we consider a local net of $C^{*}$-algebras $(\mathfrak{A}, U)$ acting on a Hilbert space $\mathcal{H}$. However, we do not assume that $\mathcal{H}$ contains the vacuum vector or non-trivial single-particle subspaces $\mathcal{H}_{ \pm}$. To study particle aspects in this general situation we use the theory of particle weights $[10,16,41,42]$ which we recall in this and the next subsection. With the help of this theory we formulate in Definitions 2.7 and 2.12 below the central notion of this paper: superselection of direction of motion.

First, we recall two useful concepts: almost locality and the energy decreasing property. An observable $B \in \mathfrak{A}$ is called almost local, if there exists a net of operators $\left\{B_{r} \in \mathfrak{A}\left(\mathcal{O}_{r}\right) \mid r>0\right\}$, s.t. for any $k \in \mathbb{N}_{0}$,

$$
\lim _{r \rightarrow \infty} r^{k}\left\|B-B_{r}\right\|=0
$$

where $\mathcal{O}_{r}=\left\{(t, \boldsymbol{x}) \in \mathbb{R}^{2}|| t|+| \boldsymbol{x} \mid<r\right\}$. We say that an operator $B \in \mathfrak{A}$ is energy decreasing, if its energy-momentum transfer is a compact set which does not intersect with the closed forward lightcone $V_{+}$. We recall that the energy-momentum transfer (or the Arveson spectrum w.r.t. $\alpha$ ) of an observable $B \in \mathfrak{A}$ is the closure of the union of supports of the distributions

$$
\left(\Psi_{1} \mid \tilde{B}(p) \Psi_{2}\right)=(2 \pi)^{-1} \int d^{2} x e^{-i p x}\left(\Psi_{1} \mid B(x) \Psi_{2}\right)
$$

over all $\Psi_{1}, \Psi_{2} \in \mathcal{H}$, where $p=(\omega, \boldsymbol{p}), x=(t, \boldsymbol{x})$ and $p x=\omega t-\boldsymbol{p} \boldsymbol{x}$.

Following [16,41], we introduce the subspace $\mathcal{L}_{0} \subset \mathfrak{A}$, spanned by operators which are both almost local and energy decreasing, and the corresponding left ideal in $\mathfrak{A}$ :

$$
\mathcal{L}:=\left\{A B \mid A \in \mathfrak{A}, B \in \mathcal{L}_{0}\right\} .
$$

Particle weights form a specific class of sesquilinear forms on $\mathcal{L}$ :

Definition 2.6. A particle weight is a non-zero, positive sesquilinear form $\psi$ on the left ideal $\mathcal{L}$, satisfying the following conditions:

1. For any $L_{1}, L_{2} \in \mathcal{L}$ and $A \in \mathfrak{A}$ the relation $\psi\left(A L_{1}, L_{2}\right)=\psi\left(L_{1}, A^{*} L_{2}\right)$ holds.

2. For any $L_{1}, L_{2} \in \mathcal{L}$ and $x \in \mathbb{R}^{2}$ the relation $\psi\left(\alpha_{x}\left(L_{1}\right), \alpha_{x}\left(L_{2}\right)\right)=\psi\left(L_{1}, L_{2}\right)$ holds.

3. For any $L_{1}, L_{2} \in \mathcal{L}$ the map $\mathbb{R}^{2} \ni x \rightarrow \psi\left(L_{1}, \alpha_{x}\left(L_{2}\right)\right)$ is continuous. Its Fourier transform is supported in a shifted lightcone $V_{+}-q$, where $q \in V_{+}$does not depend on $L_{1}, L_{2}$.

Let us now summarize the pertinent properties of particle weights established in [41] (in a slightly different framework). As a consequence of Theorem 2.9, stated below, particle weights satisfy the following clustering property [41]:

$$
\int d \boldsymbol{x}\left|\psi\left(L_{1}, \alpha_{x}\left(L_{2}\right)\right)\right|<\infty
$$

valid for $L_{1}=B_{1}^{*} A_{1} B_{1}^{\prime}, L_{2}=B_{2}^{*} A_{2} B_{2}^{\prime}$, where $B_{1}, B_{1}^{\prime}, B_{2}, B_{2}^{\prime} \in \mathcal{L}_{0}$ and $A_{1}, A_{2} \in \mathfrak{A}$ are almost local. In view of this bound, the GNS representation $\left(\pi_{\psi}, \mathcal{H}_{\pi_{\psi}}\right)$ induced by a particle weight $\psi$ is well suited for a description of physical systems which are localized in space (e.g. configurations of particles). The Hilbert space $\mathcal{H}_{\pi_{\psi}}$ is given by

$$
\mathcal{H}_{\pi_{\psi}}=(\mathcal{L} /\{L \in \mathcal{L} \mid \psi(L, L)=0\})^{\mathrm{cpl}}
$$


and the respective equivalence class of an element $L \in \mathcal{L}$ is denoted by $|L\rangle \in \mathcal{H}_{\pi_{\psi}}$. The completion is taken w.r.t. the scalar product $\left\langle L_{1} \mid L_{2}\right\rangle:=\psi\left(L_{1}, L_{2}\right)$. The representation $\pi_{\psi}$ acts on $\mathcal{H}_{\pi_{\psi}}$ as follows:

$$
\pi_{\psi}(A)|L\rangle=|A L\rangle, \quad A \in \mathfrak{A} .
$$

This representation is covariant and the translation automorphisms are implemented by the strongly continuous group of unitaries $U_{\pi_{\psi}}$, given by

$$
U_{\pi_{\psi}}(x)|L\rangle=\left|\alpha_{x}(L)\right\rangle, \quad x \in \mathbb{R}^{2}, L \in \mathcal{L}
$$

which is called the standard representation of translations in the representation $\pi_{\psi}$. By Property 3 in Definition 2.6 above, its spectrum is contained in a shifted closed forward lightcone. The corresponding canonical representation will be denoted by $U_{\pi_{\psi}}^{\mathrm{can}}$ (cf. the discussion below Definition 2.1). We also introduce operators $\left(Q^{0}, \boldsymbol{Q}\right)$ of characteristic energy-momentum of $\psi$ which are the generators of the following group of unitaries on $\mathcal{H}_{\pi_{\psi}}$

$$
U_{\pi_{\psi}}^{\text {char }}(x)=U_{\pi_{\psi}}^{\text {can }}(x) U_{\pi_{\psi}}(x)^{-1} \in \pi_{\psi}(\mathfrak{A})^{\prime},
$$

i.e. $U_{\pi_{\psi}}^{\text {char }}(x)=e^{i Q^{0} t-i Q \boldsymbol{X}}$. We call a particle weight pure, if its GNS representation is irreducible. It follows from definition (2.21) that the operator of characteristic energymomentum of such a weight is a vector $q=\left(q^{0}, \boldsymbol{q}\right) \in \mathbb{R}^{2}$. It can be interpreted as the energy and momentum of the plane wave configuration of the particle described by this weight $[2,41]$.

To extract properties of elementary subsystems (particles) of a physical system described by a given (possibly non-pure) particle weight, it is natural to study irreducible subrepresentations of its GNS representation. To ensure that there are sufficiently many such subrepresentations, we restrict attention to particle weights $\psi$ whose GNS representations $\pi_{\psi}$ are of type I with atomic center. ${ }^{3}$ (In particular, $\pi_{\psi}$ appearing in our examples in Subsect. 3.4 below belong to this family). Then, by Theorem 1.31 from Chap. V of [49], there exists a unique family of Hilbert spaces $\left(\mathfrak{H}_{\alpha}, \mathfrak{K}_{\alpha}\right)_{\alpha \in \mathbb{I}}$ and a unitary $W: \mathcal{H}_{\pi_{\psi}} \rightarrow \bigoplus_{\alpha \in \mathbb{I}}\left\{\mathfrak{H}_{\alpha} \otimes \mathfrak{K}_{\alpha}\right\}$ s.t.

$$
\begin{aligned}
W \pi_{\psi}(\mathfrak{A})^{\prime \prime} W^{-1} & =\bigoplus_{\alpha \in \mathbb{I}}\left\{B\left(\mathfrak{H}_{\alpha}\right) \otimes \mathbb{C} I\right\}, \\
W \pi_{\psi}(\mathfrak{A})^{\prime} W^{-1} & =\bigoplus_{\alpha \in \mathbb{I}}\left\{\mathbb{C} I \otimes B\left(\mathfrak{K}_{\alpha}\right)\right\} .
\end{aligned}
$$

We note that a subspace $\mathcal{K}_{\alpha, e} \subset \mathcal{H}_{\pi_{\psi}}$ carries an irreducible subrepresentation $\pi_{\alpha, e}$ of $\pi_{\psi}$, if and only if $W \mathcal{K}_{\alpha, e}=\mathfrak{H}_{\alpha} \otimes \mathbb{C} e$ for some $\alpha \in \mathbb{I}$ and $e \in \mathfrak{K}_{\alpha}$. Clearly, $\pi_{\alpha, e}$ and $\pi_{\alpha, e^{\prime}}$ are unitarily equivalent for any fixed $\alpha$ and arbitrary vectors $e, e^{\prime} \in \mathfrak{K}_{\alpha}$. Choosing in any $\mathfrak{K}_{\alpha}$ an orthonormal basis $B_{\alpha}$, we obtain

$$
\pi_{\psi}=\bigoplus_{\substack{\alpha \in \mathbb{I} \\ e \in B_{\alpha}}} \pi_{\alpha, e} .
$$

\footnotetext{
3 I.e. whose center is a direct sum of one-dimensional von Neumann algebras.
} 
It is clear from the above discussion that any irreducible subrepresentation of $\pi_{\psi}$ is unitarily equivalent to some $\pi_{\alpha, e}$ in the decomposition above.

If all the representations in the decomposition (2.24) are unitarily equivalent to some fixed vacuum representation, then we call the particle weight $\psi$ neutral. Otherwise we call $\psi$ charged. In the case of charged particle weights there may occur an interplay between the translational and internal degrees of freedom of the system which we call superselection of direction of motion. To introduce this concept, we need some terminology: Let $\mathcal{H}_{\pi_{\psi}, \mathrm{R}}$ (resp. $\mathcal{H}_{\pi_{\psi}, \mathrm{L}}$ ) be the spectral subspace of the characteristic momentum operator $\boldsymbol{Q}$ of $\psi$, corresponding to the interval $[0, \infty)$ (resp. $(-\infty, 0))$. Let $\pi$ be an irreducible subrepresentation of $\pi_{\psi}$, acting on a subspace $\mathcal{K} \subset \mathcal{H}_{\pi_{\psi}}$. Then we say that $\pi$ is right-moving (resp. left-moving), if $\mathcal{K} \neq\{0\}$ and $\mathcal{K} \subset \mathcal{H}_{\pi_{\psi}, \mathrm{R}}$ (resp. $\mathcal{K} \subset \mathcal{H}_{\pi_{\psi}, \mathrm{L}}$ ). By a suitable choice of the bases $B_{\alpha}$ one can ensure that each representation $\pi_{\alpha, e}$, appearing in decomposition (2.24), has one of these properties. (In fact, exploiting relations (2.21), (2.23), one can choose such basis vectors $e \in \mathfrak{K}_{\alpha}$ that $W^{-1}\left(\mathfrak{H}_{\alpha} \otimes \mathbb{C} e\right)$ belong to $\mathcal{H}_{\pi_{\psi}, \mathrm{R}}$ or $\mathcal{H}_{\pi_{\psi}, \mathrm{L}}$ ). After this preparation we define the central concept of the present paper:

Definition 2.7. Let $\mathcal{W}$ be a family of particle weights and assume that their GNS representations $\left\{\left(\pi_{\psi}, \mathcal{H}_{\pi_{\psi}}\right) \mid \psi \in \mathcal{W}\right\}$ are of type I with atomic centers. Suppose that for any $\psi, \psi^{\prime} \in \mathcal{W}$ the following properties hold:

1. $\pi_{\psi}$ has both left-moving and right-moving irreducible subrepresentations.

2. No right-moving, irreducible subrepresentation of $\pi_{\psi}$ is unitarily equivalent to a left-moving irreducible subrepresentation of $\pi_{\psi^{\prime}}$.

Then we say that this family of particle weights has superselected direction of motion.

Let us now relate superselection of direction of motion in the above sense to our discussion of this concept in the Introduction. For this purpose we consider a particle weight $\psi$, whose GNS representation is of type I with atomic center and acts on a separable Hilbert space $\mathcal{H}_{\pi_{\psi}}$. Making use of formula (2.24) and identifying unitarily each $\pi_{\alpha, e}$, acting on $W^{-1}\left(\mathfrak{H}_{\alpha} \otimes \mathbb{C} e\right)$, with $\pi_{\alpha}:=\pi_{\alpha, e_{0}}$ acting on $\mathcal{K}_{\alpha}:=W^{-1}\left(\mathfrak{H}_{\alpha} \otimes \mathbb{C} e_{0}\right)$ for some chosen $e_{0} \in B_{\alpha}$, we obtain

$$
\pi_{\psi}(\mathfrak{A}) \simeq \bigoplus_{\alpha \in \mathbb{I}}\left\{\pi_{\alpha}(\mathfrak{A}) \otimes \mathbb{C} I\right\},
$$

where the r.h.s. acts on $\bigoplus_{\alpha \in \mathbb{I}}\left\{\mathcal{K}_{\alpha} \otimes \mathfrak{K}_{\alpha}\right\}$. In the sense of the same identification

$$
\pi_{\psi}(\mathfrak{A})^{\prime} \simeq \bigoplus_{\alpha \in \mathbb{I}}\left\{\mathbb{C} I \otimes B\left(\mathfrak{K}_{\alpha}\right)\right\} .
$$

Now, following [42], we choose a maximal abelian von Neumann algebra $\mathcal{M}$ in $\pi_{\psi}(\mathfrak{A})^{\prime}$, containing $\left\{U_{\pi_{\psi}}^{\text {char }}(x) \mid x \in \mathbb{R}^{2}\right\}$. As a consequence of formula (2.26),

$$
\mathcal{M} \simeq \bigoplus_{\alpha \in \mathbb{I}}\left\{\mathbb{C} I \otimes \mathcal{M}_{\alpha}\right\},
$$

where $\mathcal{M}_{\alpha} \subset B\left(\mathfrak{K}_{\alpha}\right)$ are maximal abelian von Neumann subalgebras. For any such $\mathcal{M}_{\alpha}$ there exists a Borel space $\left(Z_{\alpha}, d \mu_{\alpha}\right)$ s.t. $\left(\mathcal{M}_{\alpha}, \mathfrak{K}_{\alpha}\right) \simeq\left(L^{\infty}\left(Z_{\alpha}, d \mu_{\alpha}\right), L^{2}\left(Z_{\alpha}, d \mu_{\alpha}\right)\right)$. (This fact uses separability of the Hilbert space. See Theorem II.2.2 of [20].) Adopting this identification in (2.25) and (2.26), we obtain

$$
U_{\pi_{\psi}}^{\text {char }} \simeq \bigoplus_{\alpha \in \mathbb{I}}\left\{I \otimes U_{\alpha}^{\text {char }}\right\},
$$


where $U_{\alpha}^{\text {char }}(x) \in L^{\infty}\left(Z_{\alpha}, d \mu_{\alpha}\right)$ is the operator of multiplication by (the equivalence class of $)$ the function $Z_{\alpha} \ni z \rightarrow e^{i q_{\alpha, z} x}$, where $q_{\alpha, z}=\left(q_{\alpha, z}^{0}, \boldsymbol{q}_{\alpha, z}\right) \in \mathbb{R}^{2}$. Introducing the field of representations $\left(\pi_{\alpha, z}, \mathfrak{H}_{\alpha, z}\right)_{z \in Z_{\alpha}}$ s.t. $\pi_{\alpha, z}=\pi_{\alpha}$ and $\mathfrak{H}_{\alpha, z}=\mathcal{K}_{\alpha}$ for all $z \in Z_{\alpha}$, we obtain from relation (2.25) the existence of a unitary $\tilde{W}: \mathcal{H}_{\pi_{\psi}} \rightarrow \bigoplus_{\alpha \in \mathbb{I}} \int^{\oplus} d \mu_{\alpha}(z) \mathfrak{H}_{\alpha, z}$ s.t.

$$
\tilde{W} \pi_{\psi}(\cdot) \tilde{W}^{-1}=\bigoplus_{\alpha \in \mathbb{I}} \int_{Z_{\alpha}}^{\oplus} d \mu_{\alpha}(z) \pi_{\alpha, z}(\cdot) .
$$

This is an example of decomposition (1.2), stated in the Introduction. Moreover, as a consequence of (2.28),

$$
\tilde{W} U_{\pi_{\psi}}^{\text {char }}(x) \tilde{W}^{-1}=\bigoplus_{\alpha \in \mathbb{I}} \int_{Z_{\alpha}}^{\oplus} d \mu_{\alpha}(z) e^{i q_{\alpha, z} x},
$$

where $\left\{q_{\alpha, z}\right\}_{z \in Z_{\alpha}}$ is the field of characteristic energy-momentum vectors ${ }^{4}$ of the representations $\left(\pi_{\alpha, z}, \mathfrak{H}_{\alpha, z}\right)_{z \in Z_{\alpha}}$. As we required in the Introduction, for any particle weight with superselected direction of motion, the relation $\boldsymbol{q}_{\alpha, z} \cdot \boldsymbol{q}_{\alpha^{\prime}, z^{\prime}} \leq 0$ should imply that $\pi_{\alpha, z}$ is not unitarily equivalent to $\pi_{\alpha^{\prime}, z^{\prime}}$ for almost all $z, z^{\prime}$. This is in fact the case in view of the following proposition.

Proposition 2.8. Suppose that $\psi$ belongs to a family of particle weights which has superselected direction of motion in the sense of Definition 2.7 and s.t. its GNS representation acts on a separable Hilbert space. Then $\left\{\pi_{\alpha, z}\right\}_{z \in Z_{\alpha}}$, appearing in the decomposition (2.29) of $\pi_{\psi}$, is a field of right-moving (resp. left-moving) representations, if and only if $\boldsymbol{q}_{\alpha, z} \geq 0$ (resp. $\left.\boldsymbol{q}_{\alpha, z}<0\right)$ for almost all $z \in Z_{\alpha}$.

Proof. Suppose that $\pi_{\alpha}$ is a right-moving subrepresentation of $\pi_{\psi}$, i.e. $\mathcal{K}_{\alpha} \subset \mathcal{H}_{\pi_{\psi}, \mathrm{R}}$. We recall that $\pi_{\alpha}$ coincides with $\pi_{\alpha, e_{0}}$ acting on $\mathcal{K}_{\alpha, e_{0}}=\mathcal{K}_{\alpha}$. Since every $\pi_{\alpha, e}, e \in \mathfrak{K}$, is unitarily equivalent to $\pi_{\alpha, e_{0}}$, the property of superselection of direction of motion implies that $\mathcal{K}_{\alpha, e} \subset \mathcal{H}_{\pi_{\psi}, \mathrm{R}}$ for all $e \in \mathfrak{K}$. Consequently, $\mathcal{H}_{\alpha}=W^{-1}\left(\mathfrak{H}_{\alpha} \otimes \mathfrak{K}_{\alpha}\right) \subset \mathcal{H}_{\pi_{\psi}, \mathrm{R}}$. Since the projection $P_{\alpha}$ on $\mathcal{H}_{\alpha}$ is central, this subspace is invariant under the action of $U_{\pi_{\psi}}^{\text {char }}$. Formula (2.28) gives

$$
U_{\pi_{\psi}}^{\text {char }}(x) P_{\alpha} \simeq I \otimes U_{\alpha}^{\text {char }}(x)
$$

thus the spectra of the generators of $\mathbb{R}^{2} \ni x \rightarrow U_{\alpha}^{\text {char }}(x)$ and $\mathbb{R}^{2} \ni x \rightarrow U_{\pi_{\psi}}^{\text {char }}(x) P_{\alpha}$ coincide. In particular the spectrum of the generator of space translations of $U_{\alpha}^{\text {char }}$ is contained in $[0, \infty)$. The opposite implication follows immediately from relation (2.31).

2.4. Asymptotic functionals. In this subsection we consider a concrete class of particle weights, introduced in $[9,10,41]$, which have applications in scattering theory. Their construction relies on the following result due to Buchholz (which remains valid in higher dimensions).

\footnotetext{
4 This terminology is consistent with the discussion after formula (2.21). In fact, under some technical restrictions each $\pi_{\alpha, z}$ is induced by some pure particle weight $\psi_{\alpha, z}$, whose characteristic energy-momentum vector is $q_{\alpha, z}[41,42]$, cf. also formula (1.3).
} 
Theorem 2.9 ([10]). Let $(\mathfrak{A}, U)$ be a local net of $C^{*}$-algebras on $\mathbb{R}^{2}$. Then, for any $E \geq 0, L \in \mathcal{L}$,

$$
\left\|P_{E} \int_{K} d \boldsymbol{x}\left(L^{*} L\right)(\boldsymbol{x}) P_{E}\right\| \leq c
$$

where $P_{E}$ is the spectral projection on vectors of energy not larger than $E, K \subset \mathbb{R}$ is a compact interval, and $c$ is a constant independent of $K$.

Following [41], we introduce the algebra of detectors $\mathcal{C}=\operatorname{span}\left\{L_{1}^{*} L_{2}: L_{1}, L_{2} \in \mathcal{L}\right\}$ and equip it with a locally convex topology, given by the family of seminorms

$$
p_{E}(C)=\sup \left\{\int d \boldsymbol{x}|(\Psi \mid C(\boldsymbol{x}) \Psi)| \mid \Psi \in P_{E} \mathcal{H},\|\Psi\| \leq 1\right\}, \quad C \in \mathcal{C},
$$

labelled by $E \geq 0$, which are finite by Theorem 2.9. Next, for any $\Psi \in \mathcal{H}$ of bounded energy, (i.e. belonging to $P_{E} \mathcal{H}$ for some $E \geq 0$ ), we define a sequence of functionals $\left\{\sigma_{\Psi}^{(T)}\right\}_{T \in \mathbb{R}}$ from the topological dual of $\mathcal{C}$ :

$$
\sigma_{\Psi}^{(T)}(C):=\int d t h_{T}(t) \int d \boldsymbol{x}(\Psi \mid C(t, \boldsymbol{x}) \Psi), \quad C \in \mathcal{C}
$$

As this sequence is uniformly bounded in $T$ w.r.t. any seminorm $p_{E}$, the Alaoglu-Bourbaki theorem gives limit points $\sigma_{\Psi}^{\text {out }} \in \mathcal{C}^{*}$ as $T \rightarrow \infty$, which are called the asymptotic functionals. The following fact was shown in [41]:

Proposition 2.10 ([41]). If $\sigma_{\Psi}^{\text {out }} \neq 0$, then the sesquilinear forms on $\mathcal{L}$, given by

$$
\psi_{\Psi}^{\text {out }}\left(L_{1}, L_{2}\right):=\sigma_{\Psi}^{\text {out }}\left(L_{1}^{*} L_{2}\right),
$$

are particle weights, in the sense of Definition 2.6.

Fundamental results from [2] suggest a physical interpretation of the particle weights $\psi_{\Psi}^{\text {out }}$ as mixtures of plane wave configurations of all the particle types described by the theory (cf. formulas (1.2), (1.3)). Accordingly, we say that a given theory has a nontrivial particle content, if it admits some non-zero asymptotic functionals $\sigma_{\Psi}^{\text {out }}$. This is the case in any massless two-dimensional theory of Wigner particles (in a vacuum representation) as a consequence of the following theorem. A proof of this statement, which is our main technical result, is given in Appendix A.

Theorem 2.11. Let $(\mathfrak{A}, U)$ be a local net of $C^{*}$-algebras on $\mathbb{R}^{2}$ in a vacuum representation, acting on a Hilbert space $\mathcal{H}$. Then, for any $\Psi \in P_{E} \mathcal{H}^{\text {out }}, E \geq 0$,

$$
\begin{aligned}
\psi_{\Psi}^{\text {out }}\left(L_{1}, L_{2}\right) & =\lim _{T \rightarrow \infty} \int d t h_{T}(t) \int d \boldsymbol{x}\left(\Psi \mid\left(L_{1}^{*} L_{2}\right)(t, \boldsymbol{x}) \Psi\right) \\
& =\int d \boldsymbol{x}\left(\rho_{+, \Psi}+\rho_{-, \Psi}\right)\left(\left(L_{1}^{*} L_{2}\right)(\boldsymbol{x})\right),
\end{aligned}
$$

where the functionals $\rho_{ \pm} \Psi$ are defined by (2.11), (2.12). In particular, $\psi_{\Psi}^{\text {out }}=0$, if and only if $\Psi \in \mathbb{C} \Omega$. 
In a theory of Wigner particles $\mathbb{C} \Omega \neq \mathcal{H}_{ \pm} \subset \mathcal{H}^{\text {out }}$, thus the particle content is non-trivial by the above result. However, non-zero asymptotic functionals may also appear in the absence of Wigner particles, i.e. when one or both of the subspaces $\mathcal{H}_{ \pm}$equal $\mathcal{H}_{+} \cap \mathcal{H}_{-}$. If this is the case, then we say that the net $(\mathfrak{A}, U)$ describes infraparticles. Theorem 3.6 below provides a large class of such theories. In Theorem 3.10 we show that some of these models describe excitations whose direction of motion is superselected in the following sense:

Definition 2.12. Let (A, $U$ ) be a net describing infraparticles. We say that the infraparticles of the net $(\mathfrak{A}, U)$ have superselected direction of motion, if $\left\{\psi_{\Psi}^{\text {out }} \mid \Psi \neq 0, \Psi \in\right.$ $\left.P_{E} \mathcal{H}, E \geq 0\right\}$ is a family of particle weights with superselected direction of motion in the sense of Definition 2.7.

\section{Particle Aspects of Conformal Field Theories}

3.1. Preliminaries. In this section we are interested in particle aspects of chiral conformal field theories. To emphasize the relevant properties of these models, we base our investigation on the concept of a local net of von Neumann algebras on $\mathbb{R}$, defined below. There are many examples of such nets. In particular, they arise from Möbius covariant nets on $S^{1}$ by means of the Cayley transform and the subsequent restriction to the real line. The simplest example is the so-called $U(1)$-current net [15], whose subnets and extensions are well-studied. For certain classes of nets on $S^{1}$ even classification results have been obtained [36,37].

Definition 3.1. A local net of von Neumann algebras on $\mathbb{R}$ is a pair $(\mathcal{A}, V)$ consisting of a map $\mathcal{I} \rightarrow \mathcal{A}(\mathcal{I})$ from the family of open, bounded subsets of $\mathbb{R}$ to the family of von Neumann algebras on a Hilbert space $\mathcal{K}$ and a strongly continuous unitary representation of translations $\mathbb{R} \ni s \rightarrow V(s)$, acting on $\mathcal{K}$, which are subject to the following conditions:

1. (isotony) If $\mathcal{I} \subset \mathfrak{J}$, then $\mathcal{A}(\mathcal{I}) \subset \mathcal{A}(\mathfrak{J})$.

2. (locality) If $\mathcal{I} \cap \mathfrak{J}=\varnothing$, then $[\mathcal{A}(\mathcal{I}), \mathcal{A}(\mathfrak{J})]=0$.

3. (covariance) $V(s) \mathcal{A}(\mathcal{I}) V(s)^{*}=\mathcal{A}(\mathcal{I}+s)$ for any $s \in \mathbb{R}$.

4. (positivity of energy) The spectrum of $V$ coincides with $\mathbb{R}_{+}$.

We also denote by $\mathcal{A}$ the quasilocal $C^{*}$-algebra of this net, i.e. $\mathcal{A}=\overline{\bigcup_{\mathcal{I} \subset \mathbb{R}} \mathcal{A}(\mathcal{I})}$.

Since we assumed that $\mathcal{A}(\mathcal{I})$ are von Neumann algebras, we cannot demand norm continuity of the functions $s \rightarrow \beta_{s}(A), A \in \mathcal{A}$, where $\beta_{s}(\cdot)=V(s) \cdot V(s)^{*}$. This regularity property holds, however, on the following weakly dense subnet of $C^{*}$-algebras:

$$
\mathcal{I} \rightarrow \overline{\mathcal{A}}(\mathcal{I}):=\left\{A \in \mathcal{A}(\mathcal{I}) \mid \lim _{s \rightarrow 0}\left\|\beta_{s}(A)-A\right\|=0\right\} .
$$

The corresponding quasilocal algebra is denoted by $\overline{\mathcal{A}}$.

If $V$ has a unique (up to a phase) invariant, unit vector $\Omega_{0} \in \mathcal{K}$ and $\Omega_{0}$ is cyclic under the action of any $\mathcal{A}(\mathcal{I})$ (the Reeh-Schlieder property) then we say that the net $(\mathcal{A}, V)$ is in a vacuum representation. In this case $\mathcal{A}$ acts irreducibly on $\mathcal{K}$. In the course of our analysis we will also consider other representations of $(\mathcal{A}, V)$. We say that a representation $\pi: \mathcal{A} \rightarrow B\left(\mathcal{K}_{\pi}\right)$ is covariant, if there exists a strongly continuous group of unitaries $V_{\pi}$ on $\mathcal{K}_{\pi}$, s.t.

$$
\pi\left(\alpha_{s}(A)\right)=V_{\pi}(s) \pi(A) V_{\pi}(s)^{*}, \quad A \in \mathcal{A}, s \in \mathbb{R} .
$$


Moreover, we say that this representation has positive energy, if the spectrum of $V_{\pi}$ coincides with $\mathbb{R}_{+}$. If $\pi$ is locally normal (i.e. its restriction to any local algebra $\mathcal{A}(\mathcal{I})$ is normal) then $\left(\pi(\mathcal{A}), V_{\pi}\right)$ is again a net of von Neumann algebras in the sense of Definition 3.1.

Let $\left(\mathcal{A}_{1}, V_{1}\right)$ and $\left(\mathcal{A}_{2}, V_{2}\right)$ be two nets of von Neumann algebras on $\mathbb{R}$, acting on Hilbert spaces $\mathcal{K}_{1}$ and $\mathcal{K}_{2}$. To construct a local net $(\mathfrak{A}, U)$ on $\mathbb{R}^{2}$, acting on the tensor product space $\mathcal{H}=\mathcal{K}_{1} \otimes \mathcal{K}_{2}$, we identify the two real lines with the lightlines $I_{ \pm}=\left\{(t, \boldsymbol{x}) \in \mathbb{R}^{2} \mid \boldsymbol{x} \mp t=0\right\}$ in $\mathbb{R}^{2}$. We first specify the unitary representation of translations

$$
U(t, \boldsymbol{x}):=V_{1}\left(\frac{1}{\sqrt{2}}(t-\boldsymbol{x})\right) \otimes V_{2}\left(\frac{1}{\sqrt{2}}(t+\boldsymbol{x})\right),
$$

whose spectrum is easily seen to coincide with $V_{+}$as a consequence of Property 4 from Definition 3.1. We mention for future reference that if $\alpha_{(t, x)}(\cdot):=U(t, \boldsymbol{x}) \cdot U(t, \boldsymbol{x})^{*}$ is the corresponding group of translation automorphisms and $\beta_{s}^{(1 / 2)}(\cdot):=V_{1 / 2}(s)$. $V_{1 / 2}(s)^{*}$, then

$$
\alpha_{(t, x)}\left(A_{1} \otimes A_{2}\right)=\beta_{\frac{1}{\sqrt{2}}(t-x)}^{(1)}\left(A_{1}\right) \otimes \beta_{\frac{1}{\sqrt{2}}(t+x)}^{(2)}\left(A_{2}\right), \quad A_{1} \in \mathcal{A}_{1}, A_{2} \in \mathcal{A}_{2}
$$

Any double cone $D \subset \mathbb{R}^{2}$ can be expressed as a product of intervals on lightlines $D=\mathcal{I}_{1} \times \mathcal{I}_{2}$. We define the corresponding local von Neumann algebra by $\mathfrak{A}^{\mathrm{vN}}(D):=$ $\mathcal{A}_{1}\left(\mathcal{I}_{1}\right) \otimes \mathcal{A}_{2}\left(\mathcal{I}_{2}\right)$, and for a general open region $\mathcal{O}$ we put $\mathfrak{A}^{\mathrm{vN}}(\mathcal{O})=\bigvee_{D \subset \mathcal{O}} \mathfrak{A}^{\mathrm{vN}}(D)$. The net of von Neumann algebras $\left(\mathfrak{A}^{\mathrm{vN}}, U\right)$, which we call the chiral net, satisfies all the properties from Definition 2.1 except for the regularity Property 5. Therefore, we introduce the following weakly dense subnet of $C^{*}$-algebras

$$
\mathcal{O} \rightarrow \mathfrak{A}(\mathcal{O}):=\left\{A \in \mathfrak{A}^{\mathrm{vN}}(\mathcal{O}) \mid \lim _{x \rightarrow 0}\left\|\alpha_{x}(A)-A\right\|=0\right\}
$$

and denote the corresponding quasilocal algebra by $\mathfrak{A}$. Then $(\mathfrak{A}, U)$ is a local net of $C^{*}$-algebras in the sense of Definition 2.1. We will call it the regular chiral net and refer to $\left(\mathcal{A}_{1}, V_{1}\right),\left(\mathcal{A}_{2}, V_{2}\right)$ as its chiral components. We note for future reference that if $\mathfrak{A}$ acts irreducibly on $\mathcal{H}$, then $U$ is automatically the canonical representation of translations of this net (cf. Subsect. 2.1). Another useful fact is the obvious inclusion

$$
\overline{\mathcal{A}}_{1} \otimes_{\mathrm{alg}} \overline{\mathcal{A}}_{2} \subset \mathfrak{A}
$$

where $\otimes_{\text {alg }}$ is the algebraic tensor product.

Let $\left(\mathfrak{A}^{\mathrm{vN}}, U\right)$ be a chiral net, whose chiral components are $\left(\mathcal{A}_{1}, V_{1}\right)$ and $\left(\mathcal{A}_{2}, V_{2}\right)$. Let $\pi_{1}, \pi_{2}$ be locally normal, covariant, positive energy representations of the respective nets on $\mathbb{R}$. Then the chiral net of $\left(\pi_{1}\left(\mathcal{A}_{1}\right), V_{\pi_{1}}\right),\left(\pi_{2}\left(\mathcal{A}_{2}\right), V_{\pi_{2}}\right)$ is a covariant, positive energy representation of $\left(\mathfrak{A}^{\mathrm{vN}}, U\right)$, which will be denoted by $\left(\pi\left(\mathfrak{A}^{\mathrm{vN}}\right), U_{\pi}\right), \pi=\pi_{1} \otimes \pi_{2}$ and $\pi$ is called the product representation of $\pi_{1}$ and $\pi_{2}$. We note that $\left(\pi(\mathfrak{A}), U_{\pi}\right)$ is contained in the regular subnet of $\left(\pi\left(\mathfrak{A}^{\mathrm{vN}}\right), U_{\pi}\right)$. For faithful $\pi$ these two nets coincide, due to Proposition 2.3 .3 (2) of [17]. It is easily seen that $\pi$ is faithful (resp. irreducible), if $\pi_{1}$ and $\pi_{2}$ are faithful (resp. irreducible) (cf. Theorems 5.2 and 5.9 from Chap. IV of [49]). 
3.2. Vacuum representations and asymptotic completeness. A regular chiral net $(\mathfrak{A}, U)$ is in a vacuum representation, with the vacuum vector $\Omega \in \mathcal{H}$, if and only if its chiral components $\left(\mathcal{A}_{1}, V_{1}\right),\left(\mathcal{A}_{2}, V_{2}\right)$ are in vacuum representations with the respective vacuum vectors $\Omega_{1} \in \mathcal{K}_{1}, \Omega_{2} \in \mathcal{K}_{2}$ s.t. $\Omega=\Omega_{1} \otimes \Omega_{2}$ (cf. Proposition 3.5 below). In this subsection we show that any such regular chiral net has a complete particle interpretation in terms of non-interacting Wigner particles. These facts follow from our results in [25], but the argument below is more direct.

We start from the observation that the asymptotic fields have a particularly simple form in chiral theories:

Proposition 3.2. Let $\left(\mathcal{A}_{1}, V_{1}\right),\left(\mathcal{A}_{2}, V_{2}\right)$ be two local nets of von Neumann algebras in vacuum representations, with the respective vacuum vectors $\Omega_{1}, \Omega_{2}$. Then, for any $A_{1} \in \overline{\mathcal{A}}_{1}, A_{2} \in \overline{\mathcal{A}}_{2}$,

$$
\begin{aligned}
& \Phi_{+}^{\text {out } / \text { in }}\left(A_{1} \otimes A_{2}\right)=A_{1} \otimes\left(\Omega_{2} \mid A_{2} \Omega_{2}\right) I, \\
& \Phi_{-}^{\text {out } / \text { in }}\left(A_{1} \otimes A_{2}\right)=\left(\Omega_{1} \mid A_{1} \Omega_{1}\right) I \otimes A_{2} .
\end{aligned}
$$

Proof. We consider only $\Phi_{+}^{\text {out }}$, as the remaining cases are analogous. From the defining relation (2.6) and formula (3.4), we obtain

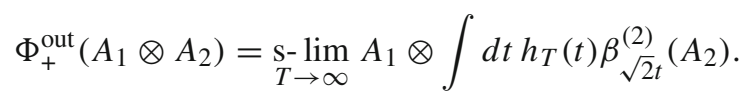

We set $A_{2}\left(h_{T}\right):=\int d t h_{T}(t) \beta_{\sqrt{2} t}^{(2)}\left(A_{2}\right)$. This sequence has the following properties:

$$
\begin{aligned}
\lim _{T \rightarrow \infty} A_{2}\left(h_{T}\right) \Omega_{2} & =\left(\Omega_{2} \mid A_{2} \Omega_{2}\right) \Omega_{2}, \\
\lim _{T \rightarrow \infty}\left\|\left[A_{2}\left(h_{T}\right), A\right]\right\| & =0, \quad \text { for any } A \in \overline{\mathcal{A}}_{2} .
\end{aligned}
$$

The first identity above follows from the mean ergodic theorem and the fact that $\Omega_{2}$ is the only vector invariant under the action of $V_{2}$. The second equality is a consequence of the locality assumption from Definition 3.1. Since $\overline{\mathcal{A}}_{2}$ acts irreducibly, any $\Psi \in \mathcal{K}_{2}$ has the form $\Psi=A \Omega_{2}$ for some $A \in \overline{\mathcal{A}}_{2}$ [45]. Thus we obtain from (3.10), (3.11),

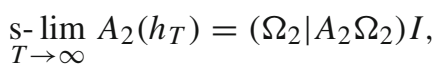

which completes the proof.

Now we can easily prove the main result of this subsection:

Theorem 3.3. Any regular chiral net $(\mathfrak{A}, U)$ in a vacuum representation is asymptotically complete. More precisely:

$$
\begin{aligned}
\mathcal{H}_{+} & =\mathcal{K}_{1} \otimes \mathbb{C} \Omega_{2}, \\
\mathcal{H}_{-} & =\mathbb{C} \Omega_{1} \otimes \mathcal{K}_{2}, \\
\mathcal{H}_{+} \text {out } \mathcal{H}_{-} & =\mathcal{H}_{+} \stackrel{\text { in }}{ } \times \mathcal{H}_{-}=\mathcal{H} .
\end{aligned}
$$

Moreover, any such theory is non-interacting.

Remark 3.4. This result and Theorem 2.11 imply the convergence of the asymptotic functional approximants $\left\{\sigma_{\Psi}^{(T)}\right\}_{T \in \mathbb{R}_{+}}$for all $\Psi \in \mathcal{H}$ of bounded energy in any regular chiral net in a vacuum representation. 
Proof. Using formula (2.5) and the cyclicity of the vacuum $\Omega$ under the action of $\mathfrak{A}$, we obtain

$$
\mathcal{H}_{ \pm}=\left[\Phi_{ \pm}^{\text {out }}(F) \Omega \mid F \in \mathfrak{A}\right]
$$

where $[\cdot]$ denotes the norm closure. Applying Proposition 3.2 and exploiting the cyclicity of $\Omega_{1 / 2}$ under the action of $\overline{\mathcal{A}}_{1 / 2}$, we obtain (3.13) and (3.14). The asymptotic completeness relation (3.15) also follows from Proposition 3.2: For any $A_{1} \in \overline{\mathcal{A}}_{1}, A_{2} \in \overline{\mathcal{A}}_{2}$,

$$
\Phi_{+}^{\text {out }}\left(A_{1} \otimes I\right) \Phi_{-}^{\text {out }}\left(I \otimes A_{2}\right) \Omega=\Phi_{+}^{\text {in }}\left(A_{1} \otimes I\right) \Phi_{-}^{\text {in }}\left(I \otimes A_{2}\right) \Omega=A_{1} \Omega_{1} \otimes A_{2} \Omega_{2} .
$$

Exploiting once again cyclicity of $\Omega_{1 / 2}$, we obtain that scattering states are dense in the Hilbert space.

Now let us show the lack of interaction: Let $\Psi_{ \pm} \in \mathcal{H}_{ \pm}$. Then, by (3.13), (3.14) and the irreducibility of the action of $\overline{\mathcal{A}}_{1 / 2}$ on $\mathcal{K}_{1 / 2}$, there exist $A_{1} \in \overline{\mathcal{A}}_{1}, A_{2} \in \overline{\mathcal{A}}_{2}$ s.t. $\Psi_{+}=A_{1} \Omega_{1} \otimes \Omega_{2}$ and $\Psi_{-}=\Omega_{1} \otimes A_{2} \Omega_{2}$. Then

$$
\begin{aligned}
\Psi_{+} \stackrel{\text { out }}{\times} \Psi_{-} & =\Phi_{+}^{\text {out }}\left(A_{1} \otimes I\right) \Phi_{-}^{\text {out }}\left(I \otimes A_{2}\right) \Omega=A_{1} \Omega_{1} \otimes A_{2} \Omega_{2} \\
& =\Phi_{+}^{\text {in }}\left(A_{1} \otimes I\right) \Phi_{-}^{\text {in }}\left(I \otimes A_{2}\right) \Omega=\Psi_{+} \times \Psi_{-} .
\end{aligned}
$$

Hence the scattering operator, defined in (2.10), equals the identity on $\mathcal{H}$.

3.3. Charged representations and infraparticles. It is the goal of this subsection to clarify the particle content of chiral conformal field theories in charged representations. More detailed particle properties of such theories, e.g. superselection of direction of motion, will be studied in the next subsection.

Let us first note the following simple relation between the single-particle subspaces of a regular chiral net and the invariant vectors of its chiral components.

Proposition 3.5. Let $\left(\mathcal{A}_{1}, V_{1}\right),\left(\mathcal{A}_{2}, V_{2}\right)$ be local nets of von Neumann algebras on $\mathbb{R}$. Then $V_{1}$ (resp. $V_{2}$ ) has a non-trivial invariant vector, if and only if the single-particle subspace $\mathcal{H}_{-}\left(\right.$resp. $\left.\mathcal{H}_{+}\right)$of the corresponding regular chiral net $(\mathfrak{A}, U)$ is non-trivial.

Proof. Suppose there exists a non-zero $\Omega_{1} \in \mathcal{K}_{1}$, invariant under the action of $V_{1}$. Then, for any $\Psi_{2} \in \mathcal{K}_{2}$,

$$
U(t,-t)\left(\Omega_{1} \otimes \Psi_{2}\right)=\Omega_{1} \otimes \Psi_{2},
$$

for $t \in \mathbb{R}$. Hence the subspace $\mathcal{H}_{-}$is non-trivial. Similarly, the existence of a non-zero $\Omega_{2} \in \mathcal{K}_{2}$, invariant under the action of $V_{2}$, implies the non-triviality of $\mathcal{H}_{+}$.

Now suppose $\Psi \in \mathcal{H}_{-}$and $V_{1}$ has no non-trivial, invariant vectors. Then, by the mean ergodic theorem,

$$
\Psi=\lim _{T \rightarrow \infty} \frac{1}{T} \int_{0}^{T} d t U(t,-t) \Psi=\lim _{T \rightarrow \infty} \frac{1}{T} \int_{0}^{T} d t\left(V_{1}(\sqrt{2} t) \otimes I\right) \Psi=0 .
$$

Thus we established that $\mathcal{H}_{-}=\{0\}$. Similarly, the absence of non-trivial, invariant vectors of $V_{2}$ implies that $\mathcal{H}_{+}=\{0\}$. 
Let $(\mathfrak{A}, U)$ be a regular chiral net in a charged irreducible (product) representation. That is $\mathfrak{A}$ acts irreducibly on a non-trivial Hilbert space, which has the tensor product structure, by our definition of chiral nets, and does not contain non-zero invariant vectors of $U$. The particle structure of such theories is described by the following theorem.

Theorem 3.6. Let $(\mathfrak{A}, U)$ be a regular chiral net in a charged irreducible (product) representation acting on a Hilbert space $\mathcal{H}$. Then:

(a) $\mathcal{H}_{+}=\{0\}$ or $\mathcal{H}_{-}=\{0\}$, i.e. the theory does not describe Wigner particles.

(b) For any non-zero vector $\Psi \in P_{E} \mathcal{H}, E \geq 0$, all the limit points of the net $\left\{\sigma_{\Psi}^{(T)}\right\}_{T \in \mathbb{R}_{+}}$, given by (2.34), are different from zero.

Hence $(\mathfrak{A}, U)$ describes infraparticles.

Proof. Part (a) follows immediately from Proposition 3.5 and the absence of non-zero invariant vectors of $U$ in $\mathcal{H}$. As for part (b), since $\mathfrak{A}$ acts irreducibly on $\mathcal{H}$, its chiral components $\left(\mathcal{A}_{1 / 2}, V_{1 / 2}\right)$ act irreducibly on their respective Hilbert spaces $\mathcal{K}_{1 / 2}$. We note that for any non-zero vector $\Psi \in P_{E} \mathcal{H}$ we can find a sequence of vectors $\left\{\Psi_{n}\right\}_{n \in \mathbb{N}}$ from $\mathcal{K}_{1}$ s.t. $\Psi_{1} \neq 0$ and

$$
(\Psi \mid(C \otimes I) \Psi)=\sum_{n \in \mathbb{N}}\left(\Psi_{n} \mid C \Psi_{n}\right)
$$

for all $C \in B\left(\mathcal{K}_{1}\right)$. Moreover, we can assume without loss of generality that $\mathcal{K}_{1}$ does not contain non-trivial invariant vectors of $V_{1}$. Then we obtain from Lemma A.1 (b) the existence of a local operator $A \in \mathcal{A}_{1}$ and $f \in S(\mathbb{R})$ s.t. supp $\tilde{f} \cap \mathbb{R}_{+}=\varnothing$, which satisfy $A(f) \Psi_{1} \neq 0$. We note that any $B:=A(f) \otimes I$ is a non-zero element of $\mathfrak{A}$ which is almost local and energy decreasing. Consequently, $B^{*} B$ belongs to the algebra of detectors $\mathcal{C}$ of the net $(\mathfrak{A}, U)$. We consider the corresponding asymptotic functional approximants

$$
\begin{aligned}
\sigma_{\Psi}^{(T)}\left(B^{*} B\right) & =\int d t h_{T}(t) \int d \boldsymbol{x}\left(\Psi \mid \alpha_{(t, \boldsymbol{x})}\left(B^{*} B\right) \Psi\right) \\
& \left.=\int d t h_{T}(t) \int d \boldsymbol{x}\left(\Psi \mid \beta_{(\sqrt{2})^{-1}(t-x)}^{(1)}\left(A(f)^{*} A(f)\right) \otimes I\right) \Psi\right) \\
& \geq \int d \boldsymbol{x}\left(\Psi_{1} \mid\left(\beta_{(\sqrt{2})^{-1} \boldsymbol{x}}^{(1)}\left(A(f)^{*} A(f)\right)\right) \Psi_{1}\right) \neq 0,
\end{aligned}
$$

where in the last step we made use of (3.21). As the last expression is independent of $T$, all the limit points of $\left\{\sigma_{\Psi}^{(T)}\right\}_{T \in \mathbb{R}_{+}}$are different from zero.

3.4. Infraparticles with superselected direction of motion. In Theorem 3.6 above we have shown that any charged irreducible (product) representation of a chiral conformal field theory contains infraparticles. In this subsection we demonstrate that in a large class of examples these infraparticles have superselected direction of motion in the sense of Definition 2.12.

Let $(\mathcal{A}, V)$ be a local net of von Neumann algebras on $\mathbb{R}$, acting on a Hilbert space $\mathcal{K}$. We assume that this net is in a vacuum representation, with the vacuum vector $\Omega_{0} \in \mathcal{K}$. Let $W$ be a unitary operator on $\mathcal{K}$ which implements a symmetry of this net, i.e.

$$
\begin{aligned}
W \mathcal{A}(\mathcal{I}) W^{*} & \subset \mathcal{A}(\mathcal{I}), \\
W V(t) W^{*} & =V(t), \\
W \Omega_{0} & =\Omega_{0},
\end{aligned}
$$


for any open, bounded interval $\mathcal{I} \subset \mathbb{R}$ and any $t \in \mathbb{R}$. We assume that $W$ gives rise to a non-trivial representation of the group $\mathbb{Z}_{2}$, i.e. $\operatorname{Ad} W \neq \mathrm{id}$ and $W^{2}=I$. We define the subspaces

$$
\begin{aligned}
\mathcal{A}_{\mathrm{ev}}(\mathcal{I}) & =\left\{A \in \mathcal{A}(\mathcal{I}) \mid W A W^{*}=A\right\}, \\
\mathcal{A}_{\text {odd }}(\mathcal{I}) & =\left\{A \in \mathcal{A}(\mathcal{I}) \mid W A W^{*}=-A\right\} .
\end{aligned}
$$

Let $\mathcal{A}_{\mathrm{ev}}$ (resp. $\mathcal{A}_{\mathrm{odd}}$ ) be the norm-closed linear span of all operators from some $\mathcal{A}_{\mathrm{ev}}(\mathcal{I})$ (resp. $\left.\mathcal{A}_{\text {odd }}(\mathcal{I})\right), \mathcal{I} \subset \mathbb{R}$. Clearly, $\left(\mathcal{A}_{\mathrm{ev}}, V\right)$ is again a local net of von Neumann algebras on the real line acting on $\mathcal{K}$. We introduce the subspaces $\mathcal{K}_{\mathrm{ev}}=\left[\mathcal{A}_{\mathrm{ev}} \Omega_{0}\right], \mathcal{K}_{\mathrm{odd}}=$ $\left[\mathcal{A}_{\text {odd }} \Omega_{0}\right]$, where $[\cdot]$ denotes the closure, which are invariant under the action of $\mathcal{A}_{\mathrm{ev}}$ and $V$, and satisfy $\mathcal{K}=\mathcal{K}_{\text {ev }} \oplus \mathcal{K}_{\text {odd }}$. $\mathcal{K}_{\text {odd }}$ gives rise to the representation

$$
\begin{aligned}
\pi_{\text {odd }}(A) & =\left.A\right|_{\mathcal{K}_{\text {odd }}}, \quad A \in \mathcal{A}_{\mathrm{ev}}, \\
V_{\text {odd }}(t) & =\left.V(t)\right|_{\mathcal{K}_{\text {odd }}}, \quad t \in \mathbb{R} .
\end{aligned}
$$

Its relevant properties are summarized in the following lemma, which we prove in Appendix B.

Lemma 3.7. ( $\left.\pi_{\mathrm{odd}}, \mathcal{K}_{\mathrm{odd}}\right)$ is a covariant, positive energy representation of $\left(\mathcal{A}_{\mathrm{ev}}, V\right)$, in which the translation automorphisms are implemented by $V_{\text {odd }}$ Moreover:

(a) $\pi_{\mathrm{odd}}$ is a locally normal, faithful and irreducible representation of $\mathcal{A}_{\mathrm{ev}}$.

(b) $V_{\text {odd }}$ does not admit non-trivial invariant vectors.

We set $\hat{\mathcal{A}}:=\pi_{\text {odd }}\left(\mathcal{A}_{\mathrm{ev}}\right), \hat{V}(t):=V_{\text {odd }}(t)$. By the above lemma $(\hat{\mathcal{A}}, \hat{V})$ is again a local net of von Neumann algebras on the real line. We define its representation on $\mathcal{K}_{\mathrm{ev}}$,

$$
\begin{aligned}
\pi_{\mathrm{ev}}(\hat{A}) & =\left.\pi_{\mathrm{odd}}^{-1}(\hat{A})\right|_{\mathcal{K}_{\mathrm{ev}}}, \quad \hat{A} \in \hat{\mathcal{A}}, \\
V_{\mathrm{ev}}(t) & =\left.V(t)\right|_{\mathcal{K}_{\mathrm{ev}}}, \quad t \in \mathbb{R},
\end{aligned}
$$

and state the following fact, whose proof is given in Appendix B.

Lemma 3.8. $\left(\pi_{\mathrm{ev}}, \mathcal{K}_{\mathrm{ev}}\right)$ is a covariant, positive energy representation of $(\hat{\mathcal{A}}, \hat{V})$, in which the translation automorphisms are implemented by $V_{\mathrm{ev}}$. Moreover

(a) $\pi_{\mathrm{ev}}$ is a locally normal, faithful and irreducible representation of $\hat{\mathcal{A}}$.

(b) $V_{\mathrm{ev}}$ admits a unique (up to a phase) invariant vector, which is cyclic for any $\pi_{\mathrm{ev}}(\hat{\mathcal{A}}(\mathcal{I}))$.

We conclude that $\left(\pi_{\mathrm{ev}}(\hat{\mathcal{A}}), V_{\mathrm{ev}}\right)$ is a local net of von Neumann algebras in a vacuum representation with the vacuum vector $\Omega_{0} \in \mathcal{K}_{\mathrm{ev}}$.

We remark that the above abstract construction can be performed in a number of concrete cases. If a Möbius covariant net $\mathcal{I} \rightarrow \mathcal{A}(\mathcal{I})$ on $S^{1}$, in a vacuum representation, admits an automorphism ${ }^{5} \gamma$ of order 2 which preserves the vacuum state, then one can define $W$ by

$$
W A \Omega_{0}=\gamma(A) \Omega_{0}, \quad A \in \mathcal{A}(\mathcal{I}) .
$$

\footnotetext{
5 An automorphism $\gamma$ of a net $\mathcal{A}$ is an automorphism of the quasilocal algebra $\mathcal{A}$ which preserves each local algebra $\mathcal{A}(\mathcal{I})$.
} 
This does not depend on the choice of the interval $\mathcal{I}$ and defines a unitary operator thanks to the invariance of the vacuum state. This $W$ automatically commutes with the action of the Möbius group (in particular with the action of translations) as a consequence of the Bisognano-Wichmann property [31]. Thus, upon restriction to the real line, we obtain a local net equipped with a unitary $W$ which satisfies (3.23)-(3.25). Non-trivial automorphisms $\gamma$ appear, in particular, in the $U(1)$-current net $(\gamma: J(z) \rightarrow-J(z))$ [15], in loop group nets of a compact group $G$ with a $\mathbb{Z}_{2}$-subgroup in $G$ [51] and in the tensor product net $\mathcal{A} \otimes \mathcal{A}$ for an arbitrary Möbius covariant net $\mathcal{A}$, where $\gamma$ is the flip symmetry.

Coming back to the abstract setting, we introduce the class of two-dimensional theories, we are interested in: Let $\left(\mathcal{A}_{1}, V_{1}\right),\left(\mathcal{A}_{2}, V_{2}\right)$ be two local nets of von Neumann algebras on $\mathbb{R}$, in vacuum representations, acting on Hilbert spaces $\mathcal{K}_{1}, \mathcal{K}_{2}$. We denote the respective vacuum vectors by $\Omega_{1}, \Omega_{2}$ and introduce the corresponding regular chiral net $(\mathfrak{A}, U)$. We assume the existence of unitaries $W_{1}, W_{2}$, which give rise to non-trivial representations of $\mathbb{Z}_{2}$ and implement symmetries of the respective nets on $\mathbb{R}$ as defined in (3.23)-(3.25). By the construction described above we obtain the nets $\left(\hat{\mathcal{A}}_{1}, \hat{V}_{1}\right),\left(\hat{\mathcal{A}}_{2}, \hat{V}_{2}\right)$, acting on $\mathcal{K}_{1 \text {,odd }}, \mathcal{K}_{2 \text {,odd }}$. We denote by $\left(\hat{\mathfrak{A}}^{\mathrm{vN}}, \hat{U}\right)$ the corresponding chiral net acting on $\hat{\mathcal{H}}=\mathcal{K}_{1, \text { odd }} \otimes \mathcal{K}_{2 \text {,odd }}$ and by $(\hat{\mathfrak{A}}, \hat{U})$ its regular subnet. Let us summarize its properties.

Proposition 3.9. The regular chiral net $(\hat{\mathfrak{A}}, \hat{U})$, whose chiral components are $\left(\hat{\mathcal{A}}_{1}, \hat{V}_{1}\right)$, $\left(\hat{\mathcal{A}}_{2}, \hat{V}_{2}\right)$, has the following properties:

(a) $\hat{\mathfrak{A}}$ acts irreducibly on $\hat{\mathcal{H}}$.

(b) ( $\hat{\mathfrak{A}}, \hat{U})$ does not admit Wigner particles $\left(\hat{\mathcal{H}}_{ \pm}=\{0\}\right)$, but all the asymptotic functionals of the form $\left\{\psi_{\Psi}^{\text {out }} \mid \Psi \neq 0, \Psi \in P_{E} \hat{\mathcal{H}}, E \geq 0\right\}$ are non-zero.

(c) $\pi_{\mathrm{R}}=\iota_{1} \otimes \pi_{2, \mathrm{ev}}$ and $\pi_{\mathrm{L}}=\pi_{1, \mathrm{ev}} \otimes \iota_{2}$ are irreducible, faithful, covariant representations of $(\hat{\mathfrak{A}}, \hat{U})$, acting on $\mathcal{H}_{\pi_{\mathrm{R}}}:=\mathcal{K}_{1, \text { odd }} \otimes \mathcal{K}_{2 \text {, ev }}$ and $\mathcal{H}_{\pi_{\mathrm{L}}}:=\mathcal{K}_{1, \mathrm{ev}} \otimes \mathcal{K}_{2, \text { odd }}$, respectively. The respective (canonical) unitary representations of translations are given by $U_{\pi_{\mathrm{R}}}(x):=\left.U(x)\right|_{\mathcal{H}_{\pi_{\mathrm{R}}}}$ and $U_{\pi_{\mathrm{L}}}(x):=\left.U(x)\right|_{\mathcal{H}_{\pi_{\mathrm{L}}}}$.

(d) $\mathcal{H}_{\pi_{R},-}=\{0\}$ and $\mathcal{H}_{\pi_{\mathrm{R}},+} \neq\{0\}$ while $\mathcal{H}_{\pi_{\mathrm{L}},-} \neq\{0\}$ and $\mathcal{H}_{\pi_{\mathrm{L},+}}=\{0\}$. Consequently, $\pi_{\mathrm{R}}$ is not unitarily equivalent to $\pi_{\mathrm{L}}$.

In part (c) $\iota_{1 / 2}$ are the defining representations of $\hat{\mathcal{A}}_{1 / 2}$. Representations $\pi_{1 / 2, \mathrm{ev}}$ are defined as in (3.30), (3.31).

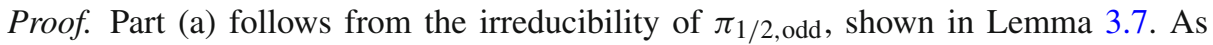
for part (b), we obtain from Lemma 3.7 (b) and Proposition 3.5 that $\hat{\mathcal{H}}_{ \pm}=\{0\}$. On the other hand, Theorem 3.6 ensures that the relevant asymptotic functionals are nonzero. Irreducibility and faithfulness of $\pi_{\mathrm{R} / \mathrm{L}}$ in part (c) follow from Lemma 3.8 (a) and Lemma 3.7 (a). Proceeding to part (d), we note that, by faithfulness of $\pi_{R}$, the net $\left(\pi_{\mathrm{R}}(\hat{\mathfrak{A}}), U_{\pi_{\mathrm{R}}}\right)$ coincides with the regular chiral subnet of $\left(\pi_{\mathrm{R}}\left(\hat{\mathfrak{A}}^{\mathrm{vN}}\right), U_{\pi_{\mathrm{R}}}\right)$, whose chiral components are $\left(\hat{\mathcal{A}}_{1}, \hat{V}_{1}\right)$ and $\left(\pi_{2, \mathrm{ev}}\left(\hat{\mathcal{A}}_{2}\right), V_{2, \text { ev }}\right)$. From Lemma 3.7 (b), Lemma 3.8 (b) and Proposition 3.5 we obtain that $\mathcal{H}_{\pi_{R},-}=\{0\}$ and $\mathcal{H}_{\pi_{R},+} \neq\{0\}$. An analogous reasoning, applied to $\pi_{\mathrm{L}}$, shows that $\mathcal{H}_{\pi_{\mathrm{L}},-} \neq\{0\}$ and $\mathcal{H}_{\pi_{\mathrm{L}},+}=\{0\}$. Hence, due to relation (2.3), the two nets are not unitarily equivalent.

In view of part (b) of the above proposition, the theory ( $\hat{\mathfrak{A}}, \hat{U})$ describes infraparticles. In the following theorem, which is our main result, we show that these infraparticles have superselected direction of motion, in the sense of Definition 2.12. 
Theorem 3.10. Consider the regular chiral net ( $\hat{\mathfrak{A}}, \hat{U})$, constructed above. Let $\psi \in$ $\left\{\psi_{\Psi}^{\text {out }} \mid \Psi \neq 0, \Psi \in P_{E} \hat{\mathcal{H}}, E \geq 0\right\}$ and let $\pi_{\psi}$ be its GNS representation. Then $\pi_{\psi}$ is a type I representation with atomic center. It contains both right-moving and left-moving irreducible subrepresentations which are unitarily equivalent to $\pi_{\mathrm{R}}$ and $\pi_{\mathrm{L}}$, respectively. Hence the theory describes infraparticles with superselected direction of motion.

Remark 3.11. Let us consider the regular chiral net ( $\mathfrak{A}, U)$ in the vacuum representation. Then, similarly as in the theorem above, the GNS representation $\pi_{\psi}$ induced by any particle weight $\psi \in\left\{\psi_{\Psi}^{\text {out }} \mid \Psi \notin \mathbb{C} \Omega, \Psi \in P_{E} \mathcal{H}, E \geq 0\right\}$ is of type I with atomic center. However, any non-trivial irreducible subrepresentation of $\pi_{\psi}$ is unitarily equivalent to the defining vacuum representation, i.e. $\psi$ is neutral. These facts are easily verified by modifying the proof below.

Proof. Let us first consider the regular chiral net ( $\mathfrak{A}, U$ ) acting on $\mathcal{H}$. By Theorem 3.3, $\mathcal{K}_{+}:=\mathcal{K}_{1, \text { odd }} \otimes \mathbb{C} \Omega_{2} \subset \mathcal{H}_{+}$and $\mathcal{K}_{-}:=\mathbb{C} \Omega_{1} \otimes \mathcal{K}_{2, \text { odd }} \subset \mathcal{H}_{-}$. Any vector $\Psi \in$

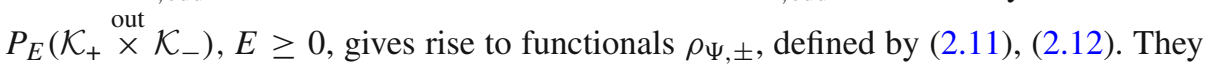
have the form

$$
\rho_{ \pm, \Psi}(\cdot)=\sum_{n \in \mathbb{N}}\left(\Psi_{ \pm, n} \mid \cdot \Psi_{ \pm, n}\right)
$$

where $\Psi_{ \pm, n} \in P_{E} \mathcal{K}_{ \pm}$(cf. formula (2.13) and the subsequent discussion). Since $\Psi \neq 0$, we can assume that $\Psi_{+, 1} \neq 0$ and $\Psi_{-, 1} \neq 0$. We also note for future reference that $\mathcal{K}_{+} \subset \mathcal{H}_{\pi_{\mathrm{R}}}$ and $\mathcal{K}_{-} \subset \mathcal{H}_{\pi_{\mathrm{L}}}$.

Let us now proceed to the net $(\hat{\mathfrak{A}}, \hat{U})$, acting on $\hat{\mathcal{H}}=\mathcal{K}_{+}{ }^{\text {out }} \times \mathcal{K}_{-} \subset \mathcal{H}$, and let $\hat{\mathcal{L}}$ be the left ideal of $\hat{\mathfrak{A}}$, given by definition (2.16). For any $\hat{L} \in \hat{\mathcal{L}}$ we define

$$
L=\left(\pi_{1, \text { odd }}^{-1} \otimes \pi_{2, \text { odd }}^{-1}\right)(\hat{L}) \in \mathcal{L}
$$

where $\mathcal{L}$ is the corresponding left ideal of $\mathfrak{A}$. We note that such $L$ leaves the subspaces $\mathcal{H}_{\pi_{\mathrm{R}}}$ and $\mathcal{H}_{\pi_{\mathrm{L}}}$ invariant. Exploiting Theorem 2.11 and formula (2.13), we obtain

$$
\psi_{\Psi}^{\text {out }}\left(\hat{L}_{1}, \hat{L}_{2}\right)=\sum_{n \in \mathbb{N}} \int d \boldsymbol{x}\left\{\left(\Psi_{+, n} \mid\left(L_{1}^{*} L_{2}\right)(\boldsymbol{x}) \Psi_{+, n}\right)+\left(\Psi_{-, n} \mid\left(L_{1}^{*} L_{2}\right)(\boldsymbol{x}) \Psi_{-, n}\right)\right\}
$$

It follows from Theorem 2.9 that for any $L$ given by (3.34) the Fourier transforms

$$
\begin{aligned}
L \tilde{\Psi}_{+, n}(\boldsymbol{p}) & :=(2 \pi)^{-1 / 2} \int d \boldsymbol{x} e^{-i \boldsymbol{p} \boldsymbol{x}} L U_{\pi_{\mathrm{R}}}(\boldsymbol{x})^{*} \Psi_{+, n}, \\
L \tilde{\Psi}_{-, n}(\boldsymbol{p}) & :=(2 \pi)^{-1 / 2} \int d \boldsymbol{x} e^{i \boldsymbol{p} \boldsymbol{x}} L U_{\pi_{\mathrm{L}}}(\boldsymbol{x})^{*} \Psi_{-, n}
\end{aligned}
$$

belong to $\mathcal{H}_{\pi_{\mathrm{R}}} \otimes L^{2}\left(\mathbb{R}_{+}, d \boldsymbol{p}\right)$ and $\mathcal{H}_{\pi_{\mathrm{L}}} \otimes L^{2}\left(\mathbb{R}_{+}, d \boldsymbol{p}\right)$ respectively. Since $\pi_{\mathrm{R}}(\hat{\mathfrak{A}})$ acts irreducibly on $\mathcal{H}_{\pi_{\mathrm{R}}}$ and $U_{\pi_{\mathrm{R}}}$ does not have non-zero invariant vectors, we obtain from Lemma A.1 (a) the existence of $\hat{L}_{+} \in \hat{\mathcal{L}}$ s.t. $L_{+} \Psi_{+, 1} \neq 0$. Since $L_{+} U_{\pi_{\mathrm{R}}}(\boldsymbol{x})^{*} \Psi_{+, 1}$ is a continuous function of $\boldsymbol{x}$, it is nonzero as a square-integrable function, hence $\left\{L_{+} \tilde{\Psi}_{+, 1}(\boldsymbol{p})\right\}_{\boldsymbol{p} \in \mathbb{R}_{+}} \neq 0$. Analogously, we can find $\hat{L}_{-} \in \hat{\mathcal{L}}$ s.t. $\left\{L_{-} \tilde{\Psi}_{-, 1}(\boldsymbol{p})\right\}_{\boldsymbol{p} \in \mathbb{R}_{+}} \neq 0$. For future reference, we note the equalities

$$
\begin{aligned}
\alpha_{x}(L) \tilde{\Psi}_{+, n}(\boldsymbol{p}) & =e^{-i(\boldsymbol{p}, \boldsymbol{p}) x} U_{\pi_{\mathrm{R}}}(x) L \tilde{\Psi}_{+, n}(\boldsymbol{p}), \\
\alpha_{x}(L) \tilde{\Psi}_{-, n}(\boldsymbol{p}) & =e^{-i(\boldsymbol{p},-\boldsymbol{p}) x} U_{\pi_{\mathrm{L}}}(x) L \tilde{\Psi}_{-, n}(\boldsymbol{p}),
\end{aligned}
$$


which hold in the sense of $\mathcal{H}_{\pi_{\mathrm{R}}} \otimes L^{2}\left(\mathbb{R}_{+}, d \boldsymbol{p}\right)$ and $\mathcal{H}_{\pi_{\mathrm{L}}} \otimes L^{2}\left(\mathbb{R}_{+}, d \boldsymbol{p}\right)$ respectively. These relations are easily verified for such $\Psi_{ \pm, n} \in P_{E} \mathcal{K}_{ \pm}$that $\mathbb{R} \ni \boldsymbol{x} \rightarrow L U_{\pi_{\mathrm{R} / \mathrm{L}}}(\boldsymbol{x})^{*} \Psi_{ \pm, n}$ decay rapidly in norm as $|\boldsymbol{x}| \rightarrow \infty$, since in this case the Fourier transform is pointwise defined. The general case follows from the fact that such vectors form a dense subspace in $P_{E} \mathcal{K}_{ \pm}$(cf. formula (3.53) below) and that the maps $P_{E} \mathcal{K}_{ \pm} \ni \Psi \rightarrow\{L \tilde{\Psi}(\boldsymbol{p})\}_{\boldsymbol{p} \in \mathbb{R}_{+}} \in$ $\mathcal{H}_{\pi_{\mathrm{R} / \mathrm{L}}} \otimes L^{2}\left(\mathbb{R}_{+}, d \boldsymbol{p}\right)$ are norm-continuous. This latter fact is a consequence of Theorem 2.9 and the (Hilbert space valued) Plancherel theorem.

After this preparation we study the structure of the GNS representation induced by $\psi$. Let us first consider the following auxiliary representation of $(\hat{\mathfrak{A}}, \hat{U})$ :

$$
\pi_{1}(\cdot):=\bigoplus_{n \in \mathbb{N}}\left(\left\{\pi_{\mathrm{R}}(\cdot) \otimes I\right\} \oplus\left\{\pi_{\mathrm{L}}(\cdot) \otimes I\right\}\right),
$$

acting on $\mathcal{H}_{\pi_{1}}:=\bigoplus_{n \in \mathbb{N}}\left(\left\{\mathcal{H}_{\pi_{\mathrm{R}}} \otimes L^{2}\left(\mathbb{R}_{+}, d \boldsymbol{p}\right)\right\} \oplus\left\{\mathcal{H}_{\pi_{\mathrm{L}}} \otimes L^{2}\left(\mathbb{R}_{+}, d \boldsymbol{p}\right)\right\}\right)$. From definition (3.40) and relation (2.22) we conclude that $\pi_{1}$ and its subrepresentations are of type I with atomic center. Moreover, $\pi_{1}$ is covariant and it is easily seen that the canonical representation of translations is given by

$$
U_{\pi_{1}}^{\mathrm{can}}(x)=\bigoplus_{n \in \mathbb{N}}\left(\left\{U_{\pi_{\mathrm{R}}}(x) \otimes I\right\} \oplus\left\{U_{\pi_{\mathrm{L}}}(x) \otimes I\right\}\right) .
$$

We note that $\pi_{\psi}$ is unitarily equivalent to a subrepresentation of $\pi_{1}$. In fact, the map $W_{1}: \mathcal{H}_{\pi_{\psi}} \rightarrow \mathcal{H}_{\pi_{1}}$, given by

$$
W_{1}:|\hat{L}\rangle \rightarrow \bigoplus_{n \in \mathbb{N}}\left(\left\{L \tilde{\Psi}_{+, n}(\boldsymbol{p})\right\}_{\boldsymbol{p} \in \mathbb{R}_{+}} \oplus\left\{L \tilde{\Psi}_{-, n}(\boldsymbol{p})\right\}_{\boldsymbol{p} \in \mathbb{R}_{+}}\right)
$$

intertwines the two representations and is an isometry by formula (3.35) and the (Hilbert space valued) Plancherel theorem. It is easily checked that the canonical representation of translations $U_{\pi_{\psi}}^{\text {can }}$ in the representation $\pi_{\psi}$ is given by the relation

$$
W_{1} U_{\pi_{\psi}}^{\mathrm{can}}(x)=U_{\pi_{1}}^{\mathrm{can}}(x) W_{1} .
$$

Recalling that $U_{\pi_{\psi}}^{\text {char }}(x)=U_{\pi_{\psi}}^{\text {can }}(x) U_{\pi_{\psi}}^{-1}(x)$, where $U_{\pi_{\psi}}$ is given by (2.20), we obtain

$$
\begin{aligned}
& W_{1} U_{\pi_{\psi}}^{\text {char }}(x)|\hat{L}\rangle \\
& =W_{1} U_{\pi_{\psi}}^{\operatorname{can}}(x)\left|\alpha_{-x}(\hat{L})\right\rangle=U_{\pi_{1}}^{\text {can }}(x) W_{1}\left|\alpha_{-x}(\hat{L})\right\rangle \\
& =\bigoplus_{n \in \mathbb{N}}\left(\left\{U_{\pi_{\mathrm{R}}}(x) \alpha_{-x}(L) \tilde{\Psi}_{+, n}(\boldsymbol{p})\right\}_{\boldsymbol{p} \in \mathbb{R}_{+}} \oplus\left\{U_{\pi_{\mathrm{L}}}(x) \alpha_{-x}(L) \tilde{\Psi}_{-, n}(\boldsymbol{p})\right\}_{\boldsymbol{p} \in \mathbb{R}_{+}}\right) \\
& =\bigoplus_{n \in \mathbb{N}}\left(\left\{I \otimes e^{i(\boldsymbol{p}, \boldsymbol{p}) x}\right\}_{\boldsymbol{p} \in \mathbb{R}_{+}} \oplus\left\{I \otimes e^{i(\boldsymbol{p},-\boldsymbol{p}) x}\right\}_{\boldsymbol{p} \in \mathbb{R}_{+}}\right) W_{1}|\hat{L}\rangle
\end{aligned}
$$

where in the last step we made use of relations (3.38), (3.39). Now let $\boldsymbol{Q}$ be the generator of space translations of $U_{\pi_{\psi}}^{\text {char }}$ and let $\mathcal{H}_{\pi_{\psi}, \mathrm{R}}\left(\right.$ resp. $\left.\mathcal{H}_{\pi_{\psi}, \mathrm{L}}\right)$ be its spectral subspace corresponding to the interval $[0, \infty)$ (resp. $(-\infty, 0))$. Then, by formula $(3.44)$,

$$
\begin{aligned}
& W_{1} \mathcal{H}_{\pi_{\psi}, \mathrm{R}}=P_{\mathrm{R}} W_{1} \mathcal{H}_{\pi_{\psi}}, \\
& W_{1} \mathcal{H}_{\pi_{\psi}, \mathrm{L}}=P_{\mathrm{L}} W_{1} \mathcal{H}_{\pi_{\psi}},
\end{aligned}
$$


where $P_{\mathrm{R} / \mathrm{L}}$ are the projections on the subspaces $\bigoplus_{n \in \mathbb{N}}\left\{\mathcal{H}_{\pi_{\mathrm{R} / \mathrm{L}}} \otimes L^{2}\left(\mathbb{R}_{+}, d \boldsymbol{p}\right)\right\}$ in $\mathcal{H}_{\pi_{1}}$. From definition (3.42) and the remarks after formula (3.37) we conclude that $P_{\mathrm{R}} W_{1} \mathcal{H}_{\pi_{\psi}} \neq\{0\}$ and $P_{\mathrm{L}} W_{1} \mathcal{H}_{\pi_{\psi}} \neq\{0\}$. Consequently $\pi_{\psi}$ has both right-moving and left-moving irreducible subrepresentations.

Let $\pi$ be an irreducible subrepresentation of $\pi_{\psi}$, acting on a non-trivial subspace $\mathcal{K} \subset \mathcal{H}_{\pi_{\psi}, \mathrm{R}}$ (i.e. a right-moving subrepresentation). Then $W_{1} \pi(\cdot) W_{1}^{*}$ is an irreducible subrepresentation of $\pi_{\mathrm{R}}(\cdot) \otimes I$ acting on $\mathcal{H}_{\pi_{\mathrm{R}}} \otimes\left(\bigoplus_{n \in \mathbb{N}} L^{2}\left(\mathbb{R}_{+}, d \boldsymbol{p}\right)\right)$. By irreducibility, we conclude that $\pi$ is unitarily equivalent to $\left(\pi_{\mathrm{R}}(\cdot) \otimes I\right) \mid \mathcal{H}_{\pi_{\mathrm{R}}} \otimes \mathbb{C} e$ for some nonzero $e \in \bigoplus_{n \in \mathbb{N}} L^{2}\left(\mathbb{R}_{+}, d \boldsymbol{p}\right)$. This latter representation can be identified with $\pi_{\mathrm{R}}$. An analogous argument shows that any left-moving irreducible subrepresentation of $\pi_{\psi}$ is unitarily equivalent to $\pi_{\mathrm{L}}$. Hence, by Proposition 3.9 (d), $(\hat{\mathfrak{A}}, \hat{U})$ describes infraparticles with superselected direction of motion.

Let us assume for a moment that $\hat{\mathcal{H}}$ is separable. Then we obtain from the above theorem and formula (2.29) that the GNS representation of any particle weight $\psi_{\Psi}^{\text {out }}$ of the net $(\hat{\mathfrak{A}}, \hat{U})$, where $\Psi \neq 0$ is a vector of bounded energy, has the form

$$
\pi_{\psi_{\Psi}^{\text {out }}} \simeq \int_{Z_{\mathrm{R}}}^{\oplus} d \mu_{\mathrm{R}}(z) \pi_{\mathrm{R}, z} \oplus \int_{Z_{\mathrm{L}}}^{\oplus} d \mu_{\mathrm{L}}(z) \pi_{\mathrm{L}, z} .
$$

Here $\left(Z_{\mathrm{R} / \mathrm{L}}, d \mu_{\mathrm{R} / \mathrm{L}}\right)$ are some Borel spaces and $\pi_{\mathrm{R} / \mathrm{L}, z}=\pi_{\mathrm{R} / \mathrm{L}}$ for all $z \in Z_{\mathrm{R} / \mathrm{L}}$. A decomposition of $\psi_{\Psi}^{\text {out }}$ into pure particle weights, which induce the irreducible representations appearing in the decomposition of $\pi_{\psi_{\Psi}^{\text {out }}}$, was obtained by Porrmann in [41,42] (cf. formula (1.3) above). However, to apply Porrmann's abstract argument, one has to restrict attention to countable (resp. separable) subsets of all the relevant objects and it is not guaranteed that the resulting (restricted) pure particle weights extend to the original domains. It is therefore worth pointing out that the theory $(\hat{\mathfrak{A}}, \hat{U})$ admits a large class of particle weights, whose decomposition can be performed in the original framework. To our knowledge this is the first such decomposition in the presence of infraparticles. (See however [35] for some partial results on the Schroer model). These particle weights belong to the set $\left\{\psi_{\Psi}^{\text {out }} \mid \Psi \in \mathcal{D}\right\}$, where $\mathcal{D} \subset \hat{\mathcal{H}}$ is a dense domain spanned by vectors of the form

$$
\Psi=F_{1} \Omega_{1} \otimes F_{2} \Omega_{2},
$$

where $F_{1} \in \mathcal{A}_{1 \text {,odd }}, F_{2} \in \mathcal{A}_{2 \text {,odd }}$ are s.t. $F_{1} \otimes I, I \otimes F_{2} \in \mathfrak{A}$ are almost local and have compact energy-momentum transfer (see formula (2.15)). The proof of the following proposition exploits some ideas from [26].

Proposition 3.12. Consider the regular chiral net $(\hat{\mathfrak{A}}, \hat{U})$ constructed above. Denote by $\hat{\mathcal{L}}$ its left ideal, given by definition (2.16). Then, for any non-zero vector $\Psi \in \mathcal{D}$, there exist continuous fields of pure particle weights $\Delta_{\mathrm{R}, n} \ni \boldsymbol{p} \rightarrow \psi_{\mathrm{R}, n, \boldsymbol{p}}(\cdot, \cdot)$ and $\Delta_{\mathrm{L}, m} \ni \boldsymbol{p} \rightarrow \psi_{\mathrm{L}, m, \boldsymbol{p}}(\cdot, \cdot)$ s.t. for any $\hat{L}_{1}, \hat{L}_{2} \in \hat{\mathcal{L}}$,

$$
\psi_{\Psi}^{\text {out }}\left(\hat{L}_{1}, \hat{L}_{2}\right)=\sum_{n \in C_{\mathrm{R}}} \int_{\Delta_{\mathrm{R}, n}} d \boldsymbol{p} \psi_{\mathrm{R}, n, \boldsymbol{p}}\left(\hat{L}_{1}, \hat{L}_{2}\right)+\sum_{m \in C_{\mathrm{L}}} \int_{\Delta_{\mathrm{L}, m}} d \boldsymbol{p} \psi_{\mathrm{L}, m, \boldsymbol{p}}\left(\hat{L}_{1}, \hat{L}_{2}\right)
$$

where $C_{\mathrm{R}}, C_{\mathrm{L}} \subset \mathbb{N}$ are non-empty finite subsets and $\Delta_{\mathrm{R}, n}, \Delta_{\mathrm{L}, m} \subset \mathbb{R}_{+}$are non-empty, open subsets for any $n \in C_{\mathrm{R}}, m \in C_{\mathrm{L}}$. Moreover: 
(a) The characteristic energy-momentum vectors of the weights $\psi_{\mathrm{R}, n, p}\left(\right.$ resp. $\left.\psi_{\mathrm{L}, m, p}\right)$ are equal to $q_{\mathrm{R}, n, \boldsymbol{p}}=(\boldsymbol{p}, \boldsymbol{p})\left(\right.$ resp. $\left.q_{\mathrm{L}, m, \boldsymbol{p}}=(\boldsymbol{p},-\boldsymbol{p})\right)$.

(b) The GNS representation induced by any $\psi_{\mathrm{R}, n, \boldsymbol{p}}$ (resp. $\left.\psi_{\mathrm{L}, m, \boldsymbol{p}}\right)$ is unitarily equivalent to $\pi_{\mathrm{R}}$ (resp. $\left.\pi_{\mathrm{L}}\right)$.

The representations $\pi_{\mathrm{R} / \mathrm{L}}$ appeared in Proposition 3.9.

Remark 3.13. Parts (b) and (d) of Proposition 3.9 show that spectral properties of the energy-momentum operators in the representations induced by the pure particle weights $\psi_{\mathrm{R} / \mathrm{L}, n, \boldsymbol{p}}$ are different from those in the original representation: In the case of $U_{\pi_{\mathrm{R}}}$ the right branch of the lightcone contains the singularities characteristic for Wigner particles, while in the left branch such singularities are absent. (For $U_{\pi_{\mathrm{L}}}$ the opposite situation occurs.) For infraparticles in physical spacetime a more radical version of this phenomenon may occur: There one expects isolated singularities at the characteristic energy-momentum values of the respective pure particle weights (cf. Sect. 2 (iii) of [16]).

Proof. Any vector $\Psi \in \mathcal{D}$ has the form

$$
\Psi=\sum_{k, l} c_{k, l} F_{\mathrm{R}, k} \Omega_{1} \otimes F_{\mathrm{L}, l} \Omega_{2},
$$

where the sum is finite and $F_{\mathrm{R}, k}, F_{\mathrm{L}, l}$ have properties specified below formula (3.48). Applying the Gram-Schmidt procedure, we can ensure that the systems of vectors $\left\{F_{\mathrm{R}, k} \Omega_{1}\right\}_{k=0}^{M},\left\{F_{\mathrm{L}, l} \Omega_{2}\right\}_{l=0}^{N}$ are orthonormal. Since $\hat{\mathcal{H}}=\mathcal{K}_{1, \text { odd }} \otimes \mathcal{K}_{2, \text { odd }} \subset \mathcal{K}_{1} \otimes \mathcal{K}_{2}=$ $\mathcal{H}$, we can write

$$
\Psi=\sum_{k, l} c_{k, l} \Phi_{+}^{\mathrm{out}}\left(F_{\mathrm{R}, k} \otimes I\right) \Phi_{-}^{\mathrm{out}}\left(I \otimes F_{\mathrm{L}, l}\right) \Omega
$$

where we made use of Proposition 3.2 applied to the net ( $\mathfrak{A}, U)$. For any $\hat{L} \in \hat{\mathcal{L}}$ we define $L=\left(\pi_{1, \text { odd }}^{-1} \otimes \pi_{2 \text {,odd }}^{-1}\right)(\hat{L}) \in \mathcal{L}$, where $\mathcal{L}$ is the left ideal of $\mathfrak{A}$, given by definition (2.16). In view of Theorem 2.11 , we get

$$
\begin{aligned}
\psi_{\Psi}^{\text {out }}\left(\hat{L}_{1}, \hat{L}_{2}\right)= & \sum_{n \in C_{\mathrm{R}}} \int d \boldsymbol{x}\left(\left(G_{\mathrm{R}, n} \otimes I\right) \Omega \mid\left(L_{1}^{*} L_{2}\right)(\boldsymbol{x})\left(G_{\mathrm{R}, n} \otimes I\right) \Omega\right) \\
& +\sum_{m \in C_{\mathrm{L}}} \int d \boldsymbol{x}\left(\left(I \otimes G_{\mathrm{L}, m}\right) \Omega \mid\left(L_{1}^{*} L_{2}\right)(\boldsymbol{x})\left(I \otimes G_{\mathrm{L}, m}\right) \Omega\right),
\end{aligned}
$$

where $G_{\mathrm{R}, n}=\sum_{k} c_{k, n} F_{\mathrm{R}, k}, G_{\mathrm{L}, m}=\sum_{l} c_{m, l} F_{\mathrm{L}, l}$ and the sets $C_{\mathrm{R}}$ and $C_{\mathrm{L}}$ are chosen so that $\Psi_{\mathrm{R}, n}:=\left(G_{\mathrm{R}, n} \otimes I\right) \Omega \neq 0$ and $\Psi_{\mathrm{L}, m}:=\left(I \otimes G_{\mathrm{L}, m}\right) \Omega \neq 0$ for $n \in C_{\mathrm{R}}$ and $m \in C_{\mathrm{L}}$. We note that both sets are non-empty, if $\Psi \neq 0$ (cf. formula (2.13) and the subsequent remarks).

Let us consider the first sum in (3.52) above: Any $L \in \mathcal{L}$ is a finite linear combination of operators of the form $A B$, where $A, B \in \mathfrak{A}$ and $B$ is almost local and energy decreasing. Since we assumed that $F_{\mathrm{R}, k} \otimes I$ are almost local, the functions

$$
\mathbb{R} \ni \boldsymbol{x} \rightarrow A B U_{\pi_{\mathrm{R}}}(\boldsymbol{x})^{*}\left(G_{\mathrm{R}, n} \otimes I\right) \Omega=A\left[B,\left(G_{\mathrm{R}, n} \otimes I\right)(-\boldsymbol{x})\right] \Omega
$$

decrease in norm faster than any inverse power of $|\boldsymbol{x}|$. Consequently, the Fourier transform

$$
L \tilde{\Psi}_{\mathrm{R}, n}(\boldsymbol{p}):=(2 \pi)^{-1 / 2} \int d \boldsymbol{x} e^{-i \boldsymbol{p} \boldsymbol{x}} L U_{\pi_{\mathrm{R}}}(\boldsymbol{x})^{*}\left(G_{\mathrm{R}, n} \otimes I\right) \Omega
$$


is a norm-continuous function. It is compactly supported in $\mathbb{R}_{+}$due to the spectrum condition and the fact that the energy-momentum transfer of each $G_{\mathrm{R}, n} \otimes I$ is bounded. By the (Hilbert space valued) Plancherel theorem, we can write

$$
\int d \boldsymbol{x}\left(\left(G_{\mathrm{R}, n} \otimes I\right) \Omega \mid\left(L_{1}^{*} L_{2}\right)(\boldsymbol{x})\left(G_{\mathrm{R}, n} \otimes I\right) \Omega\right)=\int d \boldsymbol{p}\left(L_{1} \tilde{\Psi}_{\mathrm{R}, n}(\boldsymbol{p}) \mid L_{2} \tilde{\Psi}_{\mathrm{R}, n}(\boldsymbol{p})\right) .
$$

We define

$$
\psi_{\mathrm{R}, n, \boldsymbol{p}}\left(\hat{L}_{1}, \hat{L}_{2}\right):=\left(L_{1} \tilde{\Psi}_{\mathrm{R}, n}(\boldsymbol{p}) \mid L_{2} \tilde{\Psi}_{\mathrm{R}, n}(\boldsymbol{p})\right) .
$$

It is easy to see that non-zero $\psi_{\mathrm{R}, n, \boldsymbol{p}}$ are particle weights in the sense of Definition 2.6: Positivity and Property 1 are obvious. The continuity requirement in Property 3 follows from the equality

$$
\begin{aligned}
& \psi_{\mathrm{R}, n, \boldsymbol{p}}\left(\hat{L}_{1}, \hat{L}_{2}(y)-\hat{L}_{2}\right) \\
& =(2 \pi)^{-1 / 2} \int d \boldsymbol{x} e^{-i \boldsymbol{p} \boldsymbol{x}}\left(L_{1} \tilde{\Psi}_{\mathrm{R}, n}(\boldsymbol{p}) \mid\left[\left(L_{2}(y)-L_{2}\right),\left(G_{\mathrm{R}, n} \otimes I\right)(-\boldsymbol{x})\right] \Omega\right),
\end{aligned}
$$

and from the dominated convergence theorem. Invariance under translations (Property 2) is a straightforward consequence of the formula

$$
\alpha_{x}(L) \tilde{\Psi}_{\mathrm{R}, n}(\boldsymbol{p})=e^{-i(\boldsymbol{p}, \boldsymbol{p}) x} U_{\pi_{\mathrm{R}}}(x) L \tilde{\Psi}_{\mathrm{R}, n}(\boldsymbol{p}), \quad x \in \mathbb{R}^{2} .
$$

Making use of the above relation and the spectrum condition, it is easy to see that the distribution

$$
\mathbb{R}^{2} \ni q \rightarrow(2 \pi)^{-1} \int d^{2} x e^{-i q x} \psi_{\mathrm{R}, n, \boldsymbol{p}}\left(\hat{L}_{1}, \alpha_{x}\left(\hat{L}_{2}\right)\right)
$$

is supported in $V_{+}-(\boldsymbol{p}, \boldsymbol{p})$.

Now let us show that any function $\boldsymbol{p} \rightarrow \psi_{\mathrm{R}, n, p}(\cdot, \cdot), n \in C_{\mathrm{R}}$, is non-zero on a non-empty open set $\Delta_{\mathrm{R}, n}$ : Since $\left(G_{\mathrm{R}, n} \otimes I\right) \Omega \in \mathcal{H}_{\pi_{\mathrm{R}}}$ is different from zero, $U_{\pi_{\mathrm{R}}}$ does not have non-zero invariant vectors and $\pi_{\mathrm{R}}(\hat{\mathfrak{A}})$ acts irreducibly on $\mathcal{H}_{\pi_{\mathrm{R}}}$, Lemma A.1 ensures the existence of $\hat{L} \in \hat{\mathcal{L}}$ s.t. $L\left(G_{\mathrm{R}, n} \otimes I\right) \Omega \neq 0$. Consequently, $\mathbb{R} \ni \boldsymbol{x} \rightarrow$ $L U_{\pi_{\mathrm{R}}}(\boldsymbol{x})^{*}\left(G_{\mathrm{R}, n} \otimes I\right) \Omega$ is a non-zero function and so is the norm of its Fourier transform $\mathbb{R}_{+} \ni \boldsymbol{p} \rightarrow \psi_{\mathrm{R}, n, \boldsymbol{p}}(\hat{L}, \hat{L})$. Since the functions $\mathbb{R}_{+} \ni \boldsymbol{p} \rightarrow \psi_{\mathrm{R}, n, \boldsymbol{p}}\left(\hat{L}_{1}, \hat{L}_{2}\right)$ are continuous for all $\hat{L}_{1}, \hat{L}_{2} \in \hat{\mathcal{L}}$, as we have shown above, the sets

$$
\Delta_{\mathrm{R}, n}:=\bigcup_{\hat{L}_{1}, \hat{L}_{2} \in \hat{\mathcal{L}}}\left\{\boldsymbol{p} \in \mathbb{R}_{+} \mid \psi_{\mathrm{R}, n, \boldsymbol{p}}\left(\hat{L}_{1}, \hat{L}_{2}\right) \neq 0\right\}
$$

are open and non-empty.

Let us now fix some $n \in C_{\mathrm{R}}, \boldsymbol{p} \in \Delta_{\mathrm{R}, n}$ and consider the GNS representation $\pi$ induced by $\psi_{\mathrm{R}, n, \boldsymbol{p}}$, acting on the Hilbert space $\mathcal{H}_{\pi}:=\left(\hat{\mathcal{L}} /\left\{\hat{L} \in \hat{\mathcal{L}} \mid \psi_{\mathrm{R}, n, \boldsymbol{p}}(\hat{L}, \hat{L})=0\right\}\right)^{\mathrm{cpl}}$. The equivalence class of $\hat{L} \in \hat{\mathcal{L}}$ is denoted by $|\hat{L}\rangle$ and the scalar product is given by $\left\langle\hat{L}_{1} \mid \hat{L}_{2}\right\rangle=\psi_{\mathrm{R}, n, \boldsymbol{p}}\left(\hat{L}_{1}, \hat{L}_{2}\right)$. This GNS representation has the form

$$
\begin{array}{rlrl}
\pi(\hat{A})|\hat{L}\rangle & =|\hat{A} \hat{L}\rangle, & & \hat{L} \in \hat{\mathcal{L}}, \hat{A} \in \hat{\mathfrak{A}}, \\
U_{\pi}(x)|\hat{L}\rangle & =\left|\alpha_{x}(\hat{L})\right\rangle, & \hat{L} \in \hat{\mathcal{L}}, x \in \mathbb{R}^{2},
\end{array}
$$


where $U_{\pi}$ is the standard representation of translations. We will show that $\left(\pi(\hat{\mathfrak{A}}), U_{\pi}\right)$ is unitarily equivalent to $\left(\pi_{\mathrm{R}}(\hat{\mathfrak{A}}), U_{\pi_{\mathrm{R}}}\right)$. To this end, we introduce the map $W_{\mathrm{R}}: \mathcal{H}_{\pi} \rightarrow$ $\mathcal{H}_{\pi_{\mathrm{R}}}=\mathcal{K}_{1, \text { odd }} \otimes \mathcal{K}_{2, \text { ev }}$ given by

$$
W_{\mathrm{R}}|\hat{L}\rangle=L \tilde{\Psi}_{\mathrm{R}, n}(\boldsymbol{p}), \quad \hat{L} \in \hat{\mathcal{L}} .
$$

This map is clearly an isometry. Since $\pi_{\mathrm{R}}$ acts irreducibly on $\mathcal{H}_{\pi_{\mathrm{R}}}$, we obtain that $W_{\mathrm{R}}$ has a dense range and hence it is a unitary operator. From the relation

$$
W_{\mathrm{R}} \pi(\hat{A})|\hat{L}\rangle=\pi_{\mathrm{R}}(\hat{A}) L \tilde{\Psi}_{\mathrm{R}, n}(\boldsymbol{p})=\pi_{\mathrm{R}}(\hat{A}) W_{\mathrm{R}}|\hat{L}\rangle, \quad \hat{L} \in \hat{\mathcal{L}}, \quad \hat{A} \in \hat{\mathfrak{A}}
$$

we conclude that $\pi$ and $\pi_{\mathrm{R}}$ are unitarily equivalent. Next, we obtain for any $\hat{L} \in \hat{\mathcal{L}}$ and $x \in \mathbb{R}^{2}$,

$$
U_{\pi_{\mathrm{R}}}(x) W_{\mathrm{R}}|\hat{L}\rangle=e^{i(\boldsymbol{p}, \boldsymbol{p}) x} \alpha_{x}(L) \tilde{\Psi}_{\mathrm{R}, n}(\boldsymbol{p})=e^{i(\boldsymbol{p}, \boldsymbol{p}) x} W_{\mathrm{R}} U_{\pi}(x)|\hat{L}\rangle,
$$

where in the first step we made use of relation (3.58). We recall that the spectrum of $U_{\pi_{\mathrm{R}}}$ coincides with $V_{+}$and note that $\pi(\hat{\mathfrak{A}})$ acts irreducibly on $\mathcal{H}_{\pi}$, by relation (3.64) and Proposition 3.9 (c). Thus, in view of equality (3.65),

$$
U_{\pi}^{\text {can }}(x)=e^{i(\boldsymbol{p}, \boldsymbol{p}) x} U_{\pi}(x), \quad x \in \mathbb{R}^{2}
$$

is the canonical representation of translations in the GNS representation of $\psi_{\mathrm{R}, n, \boldsymbol{p}}$. Relation (3.66) shows that $q_{\mathrm{R}, n, \boldsymbol{p}}=(\boldsymbol{p}, \boldsymbol{p})$.

The analysis of the second term on the r.h.s. of (3.52) proceeds similarly: For any $m \in C_{\mathrm{L}}$ and $\hat{L} \in \hat{\mathcal{L}}$ one introduces vectors

$$
L \tilde{\Psi}_{\mathrm{L}, m}(\boldsymbol{p}):=(2 \pi)^{-1 / 2} \int d \boldsymbol{x} e^{i \boldsymbol{p} \boldsymbol{x}} L U(\boldsymbol{x})^{*}\left(I \otimes G_{\mathrm{L}, m}\right) \Omega
$$

and functionals $\psi_{\mathrm{L}, m, \boldsymbol{p}}\left(\hat{L}_{1}, \hat{L}_{2}\right)=\left(L_{1} \tilde{\Psi}_{\mathrm{L}, m}(\boldsymbol{p}) \mid L_{2} \tilde{\Psi}_{\mathrm{L}, m}(\boldsymbol{p})\right)$. By an analogous reasoning as above one shows that for $\boldsymbol{p}$ in some non-empty, open set $\Delta_{\mathrm{L}, m} \subset \mathbb{R}_{+}$ these functionals are particle weights with characteristic energy-momentum vectors $q_{\mathrm{L}, m, \boldsymbol{p}}=(\boldsymbol{p},-\boldsymbol{p})$. Their GNS representations are unitarily equivalent to $\pi_{\mathrm{L}}$.

\section{Conclusions and Outlook}

In this work we carried out a systematic study of particle aspects of two-dimensional conformal field theories both in vacuum representations and in charged representations. In the former case we established a complete particle interpretation in terms of Wigner particles (or 'waves' in the terminology of [5]). In the latter case we proved the existence of infraparticles and verified superselection of their direction of motion in a large class of examples. We conclude that conformal field theories provide a valuable testing ground for fundamental concepts of scattering theory.

An important question which remained outside of the scope of the present work is the problem of asymptotic completeness in the case of infraparticles. We remark that the theory of particle weights offers natural formulations of this property $[9,11]$ which can be adapted to the case of massless, two-dimensional theories. We conjecture that any charged representation of a chiral conformal field theory has a complete particle interpretation in terms of infraparticles. 
A more technical circle of problems concerns the decomposition of particle weights and their representations stated in formulas (1.2), (1.3). We recall that the general procedure of [41,42] is not canonical: Firstly, it involves a choice of a maximal abelian subalgebra, acting on the representation space of the original weight. Secondly, it relies on a selection of countable subsets of all the objects involved. In view of these ambiguities it is not yet possible to associate a unique family of (infra-)particle types with any given quantum field theory. We feel that a satisfactory solution of these problems requires a systematic study of examples. A useful criterion for their classification is the type of representations induced by particle weights. Thus in the present paper we focused on representations of type I (with atomic center) which have a simple decomposition theory. Already in this elementary case we found a physically interesting phenomenon: superselection of direction of motion. It is a natural direction of further research to look for theories, whose asymptotic functionals induce representations which are not of type I with atomic center. We conjecture that such models exist and some of them describe infraparticles with superselected momentum, similar to the electron in QED.

Acknowledgements. The authors would like to thank Prof. D. Buchholz and Prof. R. Longo for interesting discussions.

Open Access This article is distributed under the terms of the Creative Commons Attribution License which permits any use, distribution, and reproduction in any medium, provided the original author(s) and the source are credited.

\section{A. Proof of Theorem 2.11}

Lemma A.1. (a) Let $(\mathfrak{A}, U)$ be a local net of $C^{*}$-algebras on $\mathbb{R}^{2}$ in the sense of Definition 2.1, acting irreducibly on a Hilbert space $\mathcal{H}$ and let $U=U^{\text {can }}$. Let $\Psi \in \mathcal{H}$ be s.t.

$$
A(f) \Psi:=\int d^{2} x \alpha_{x}(A) f(x) \Psi=0
$$

for all local operators $A \in \mathfrak{A}$ and all $f \in S\left(\mathbb{R}^{2}\right)$ s.t. supp $\tilde{f}$ is compact and supp $\tilde{f} \cap V_{+}=\varnothing$. Then $\Psi$ is invariant under the action of $U$. (Here $\tilde{f}(p):=$ $(2 \pi)^{-1} \int d^{2} x e^{i p x} f(x)$.)

(b) Let $(\mathcal{A}, V)$ be a local net of von Neumann algebras on $\mathbb{R}$ in the sense of Definition 3.1, acting irreducibly on a Hilbert space $\mathcal{K}$. Let $\Psi \in \mathcal{K}$ be s.t.

$$
A(f) \Psi:=\int d s \beta_{s}(A) f(s) \Psi=0
$$

for all local operators $A \in \mathcal{A}$ and all $f \in S(\mathbb{R})$ s.t. supp $\tilde{f}$ is compact and supp $\tilde{f} \cap \mathbb{R}_{+}=\varnothing$. Then $\Psi$ is invariant under the action of $V$. (Here $\tilde{f}(\omega):=$ $(2 \pi)^{-\frac{1}{2}} \int d s e^{i \omega s} f(s)$.)

Proof. The argument exploits some ideas from the proof of Proposition 2.1 of [13]. As for part (a), suppose that $\Psi$ is not invariant under the action of $U$. Since the map $B(\mathcal{H}) \ni A \rightarrow A(f)$ is $\sigma$-weakly continuous (cf. Lemma 5.3 of [41]) and $\mathfrak{A}$ acts irreducibly on $\mathcal{H}$, condition (A.1) implies that

$$
P\left(\Delta_{1}\right) A(f) P\left(\Delta_{2}\right) \Psi=0,
$$


where $P(\cdot)$ is the spectral measure of $U$ and $\Delta_{1}, \Delta_{2} \subset \mathbb{R}^{2}$ are open bounded sets. Since the spectrum of $U$ has Lorentz invariant lower boundary and $\Psi$ is not invariant under translations, we can choose $\Delta_{1}, \Delta_{2}$ s.t. $P\left(\Delta_{1}\right) \neq 0, P\left(\Delta_{2}\right) \Psi \neq 0$ and the closure of $\left(\Delta_{1}-\Delta_{2}\right)$ does not intersect with $V_{+}$. Choosing $f \in S\left(\mathbb{R}^{2}\right)$ s.t. supp $\tilde{f} \cap V_{+}=\varnothing$ and $\tilde{f}(p)=1$ for $p$ in the closure of $\left(\Delta_{1}-\Delta_{2}\right)$, we obtain that

$$
P\left(\Delta_{1}\right) A P\left(\Delta_{2}\right) \Psi=0
$$

for any $A \in \mathfrak{A}$. Exploiting irreducibility again, we obtain $P\left(\Delta_{1}\right)=0$, which is a contradiction. The proof of part (b) is analogous.

Lemma A.2. Let $\mathcal{K}_{ \pm} \subset \mathcal{H}_{ \pm}$be closed subspaces, invariant under the action of $U$. Let $\left\{e_{+, m}\right\}_{m \in \mathbb{I}}$ be a complete orthonormal basis in $\left(P_{E} \mathcal{K}_{+}\right)$and let $\left\{e_{-, n}\right\}_{n \in \mathbb{J}}$ be a complete orthonormal basis in $\left(P_{E} \mathcal{K}_{-}\right)$for some $E \geq 0$. Then any $\Psi \in P_{E}\left(\mathcal{K}_{+} \stackrel{\text { out }}{\times} \mathcal{K}_{-}\right)$can be expressed as

$$
\Psi=\sum_{m, n} c_{m, n} e_{+, m} \stackrel{\text { out }}{\times} e_{-, n},
$$

where $\sum_{m, n}\left|c_{m, n}\right|^{2}<\infty$.

Proof. First, we define a strongly continuous unitary representation of translations

$$
U_{0}(x)\left(\Psi_{+} \otimes \Psi_{-}\right)=\left(U(x) \Psi_{+}\right) \otimes\left(U(x) \Psi_{-}\right), \quad \Psi_{ \pm} \in \mathcal{K}_{ \pm}
$$

on $\mathcal{K}_{+} \otimes \mathcal{K}_{-}$. Then we obtain from Proposition 2.4,

$$
\Omega^{\text {out }} U_{0}(x)=U(x) \Omega^{\text {out }} \text {. }
$$

For $\Psi^{\prime}=\left(\Omega^{\text {out }}\right)^{-1} \Psi$ the above relation gives $P_{0, E} \Psi^{\prime}=\Psi^{\prime}$, where $P_{0, E}$ is the spectral projection of $U_{0}$ corresponding to the set $\left\{(\omega, \boldsymbol{p}) \in \mathbb{R}^{2} \mid \omega \leq E\right\}$. By the functional calculus, we get $P_{0, E}=P_{0, E}\left(P_{E} \otimes P_{E}\right)$. Hence

$$
\Psi^{\prime}=\left(P_{E} \otimes P_{E}\right) \Psi^{\prime}=\sum_{m, n} c_{m, n} e_{+, m} \otimes e_{-, n}
$$

By applying $\Omega^{\text {out }}$, we obtain relation (A.5).

Lemma A.3. Let $\Psi^{\prime} \in \mathcal{H}$ be a vector of bounded energy, let $F_{1}, F_{2} \in \mathfrak{A}$ be almost local and of compact energy-momentum transfer, and let $L=\sum_{k=1}^{n} A_{k} B_{k}$, where $A_{k}, B_{k} \in \mathfrak{A}$ are almost local and $B_{k}$ are, in addition, energy decreasing. Then

$$
\lim _{T \rightarrow \infty}\left(\Psi^{\prime} \mid\left[\left[Q_{T}, \Phi_{+}^{\text {out }}\left(F_{1}\right)\right], \Phi_{-}^{\text {out }}\left(F_{2}\right)\right] \Omega\right)=0
$$

where $Q_{T}=\int d t h_{T}(t) \int d \boldsymbol{x}\left(L^{*} L\right)(t, \boldsymbol{x})$. (The above sequence is well defined by Theorem 2.9.) 
Proof. First, we note that by Proposition 2.3 and Theorem 2.9,

$$
\lim _{T \rightarrow \infty}\left(\Psi^{\prime} \mid\left[\left[Q_{T}, \Phi_{+}^{\text {out }}\left(F_{1}\right)\right], \Phi_{-}^{\text {out }}\left(F_{2}\right)\right] \Omega\right)=\lim _{T \rightarrow \infty}\left(\Psi^{\prime} \mid\left[\left[Q_{T}, F_{1,+}\left(h_{T}\right)\right], F_{2,-}\left(h_{T}\right)\right] \Omega\right),
$$

if the limit on the r.h.s. exists. We introduce the auxiliary operators:

$$
Q_{ \pm, T}:=\int d t h_{T}(t) \int_{\mathbb{R}_{ \pm}} d \boldsymbol{x}\left(L^{*} L\right)(t, \boldsymbol{x})
$$

As we show below, they satisfy

$$
\lim _{T \rightarrow \infty}\left\|P_{E}\left[Q_{ \pm, T}, F_{\mp}\left(h_{T}\right)\right] P_{E}\right\|=0
$$

for any $E \geq 0$ and any $F \in \mathfrak{A}$ which is almost local and of compact energy-momentum transfer. Making use of this relation and the fact that $Q_{T}=Q_{+, T}+Q_{-, T}$, the proof is completed with the help of the Jacobi identity and Proposition 2.3 (c).

Let us now verify (A.12). As the two cases are analogous, we focus on one of them and estimate the corresponding expression as follows:

$$
\left\|P_{E}\left[Q_{-, T}, F_{+}\left(h_{T}\right)\right] P_{E}\right\| \leq \int d t d t_{1} h_{T}(t) h_{T}\left(t_{1}\right) \int_{\mathbb{R}_{-}} d \boldsymbol{x}\left\|\left[L^{*} L(t, \boldsymbol{x}), F\left(t_{1}, t_{1}\right)\right]\right\| .
$$

Since $L^{*} L$ and $F$ are almost local, we can find sequences $C_{r}, F_{r} \in \mathfrak{A}\left(\mathcal{O}_{r}\right)$, s.t. for any $n \in \mathbb{N}$ there exist $C_{n}, C_{n}^{\prime}$ s.t.

$$
\left\|L^{*} L-C_{r}\right\| \leq \frac{C_{n}}{r^{n}}, \quad\left\|F-F_{r}\right\| \leq \frac{C_{n}^{\prime}}{r^{n}} .
$$

We choose $r=\left(1+\frac{1}{4}|\boldsymbol{x}|\right)^{\varepsilon}+T^{\varepsilon}$, where $0<\varepsilon<1$ appeared in the definition of $h_{T}$. We write

$$
\begin{aligned}
{\left[\left(L^{*} L\right)(t, \boldsymbol{x}), F\left(t_{1}, t_{1}\right)\right]=} & {\left[\left(L^{*} L-C_{r}\right)(t, \boldsymbol{x}), F\left(t_{1}, t_{1}\right)\right] } \\
& +\left[C_{r}(t, \boldsymbol{x}),\left(F-F_{r}\right)\left(t_{1}, t_{1}\right)\right] \\
& +\left[C_{r}(t, \boldsymbol{x}), F_{r}\left(t_{1}, t_{1}\right)\right] .
\end{aligned}
$$

By estimates (A.14), the first two terms on the r.h.s. above give contributions to (A.13) which tend to zero in the limit $T \rightarrow \infty$. The contribution of the last term can be estimated as follows, exploiting locality,

$$
\begin{aligned}
& \int d t d t_{1} h_{T}(t) h_{T}\left(t_{1}\right) \int_{\mathbb{R}_{-}} d \boldsymbol{x}\left\|\left[C_{r}(t, \boldsymbol{x}), F_{r}\left(t_{1}, t_{1}\right)\right]\right\| \\
& \leq c \int d t d t_{1} h_{T}(t) h_{T}\left(t_{1}\right) \int_{\mathbb{R}_{-}} d \boldsymbol{x} \chi\left(\left|\boldsymbol{x}-t_{1}\right| \leq\left|t-t_{1}\right|+2 r\right),
\end{aligned}
$$

where $\chi$ is the characteristic function of the respective set and $c$ is a constant independent of $T$. Let us now derive some inequalities which hold on the support of the integrand on the r.h.s. of (A.16). First, we note that $t, t_{1} \in \operatorname{supp} h_{T}$, if and only if $t, t_{1} \in T^{\varepsilon} \operatorname{supp} h+T$, in particular $\left|t-t_{1}\right| \leq c_{1} T^{\varepsilon}$ for some $c_{1} \geq 0$. Exploiting this fact, the inequality $\left|\boldsymbol{x}-t_{1}\right| \leq\left|t-t_{1}\right|+2 r$ and the relation $r=\left(1+\frac{1}{4}|\boldsymbol{x}|\right)^{\varepsilon}+T^{\varepsilon}$, we find 
such $c_{2} \geq 0$ that $|\boldsymbol{x}| \leq c_{2} T$ and $r \leq c_{2} T^{\varepsilon}$, in particular the r.h.s. of (A.16) is finite for any $T \geq 1$. Making use of the inequalities $r \leq c_{2} T^{\varepsilon}$ and $\left|x-t_{1}\right| \leq\left|t-t_{1}\right|+2 r$, and of the fact that $t, t_{1} \in \operatorname{supp} h_{T}$, we obtain that $|x-T| \leq c_{3} T^{\varepsilon}$ for some $c_{3} \geq 0$ which implies that $\boldsymbol{x}>0$ for sufficiently large $T$. As the region of integration in the $\boldsymbol{x}$ variable is restricted to $\mathbb{R}_{-}$, we conclude that the r.h.s. of (A.16) is zero for such $T$.

Proof of Theorem 2.11. Let $Q_{T}=\int d t h_{T}(t) \int d \boldsymbol{x}\left(L^{*} L\right)(t, \boldsymbol{x})$, where $L=\sum_{k=1}^{n} A_{k} B_{k}$ is an element of the left ideal $\mathcal{L}, A_{k}$ are almost local and $B_{k} \in \mathcal{L}_{0}$. Moreover, we choose $\Psi=\Phi_{+}^{\text {out }}\left(F_{1}\right) \Phi_{-}^{\text {out }}\left(F_{2}\right) \Omega$ and $\Psi^{\prime}=\Phi_{+}^{\text {out }}\left(F_{1}^{\prime}\right) \Phi_{-}^{\text {out }}\left(F_{2}^{\prime}\right) \Omega$, where $F_{1 / 2}, F_{1 / 2}^{\prime} \in \mathfrak{A}$ are almost local and have compact energy-momentum transfer. Since $\Psi$ and $\Psi^{\prime}$ are vectors of bounded energy, we can write

$$
\begin{aligned}
\left(\Psi^{\prime} \mid Q_{T} \Phi_{+}^{\text {out }}\left(F_{1}\right) \Phi_{-}^{\text {out }}\left(F_{2}\right) \Omega\right)= & \left(\Psi^{\prime} \mid\left[\left[Q_{T}, \Phi_{+}^{\text {out }}\left(F_{1}\right)\right], \Phi_{-}^{\text {out }}\left(F_{2}\right)\right] \Omega\right) \\
& +\left(\Psi^{\prime} \mid \Phi_{-}^{\text {out }}\left(F_{2}\right) Q_{T} \Phi_{+}^{\text {out }}\left(F_{1}\right) \Omega\right) \\
& +\left(\Psi^{\prime} \mid \Phi_{+}^{\text {out }}\left(F_{1}\right) Q_{T} \Phi_{-}^{\text {out }}\left(F_{2}\right) \Omega\right) .
\end{aligned}
$$

The term with the double commutator above vanishes as $T \rightarrow \infty$ due to Lemma A.3. The second term on the r.h.s. of relation (A.17) is treated as follows:

$$
\begin{aligned}
\lim _{T \rightarrow \infty}\left(\Psi^{\prime} \mid \Phi_{-}^{\text {out }}\left(F_{2}\right) Q_{T} \Phi_{+}^{\text {out }}\left(F_{1}\right) \Omega\right) \\
=\lim _{T \rightarrow \infty}\left(\Psi^{\prime} \mid \Phi_{-}^{\text {out }}\left(F_{2}\right) \int h_{T}(t) e^{i H t} \int d \boldsymbol{x}\left(L^{*} L\right)(\boldsymbol{x}) e^{-i \boldsymbol{P} t} \Phi_{+}^{\text {out }}\left(F_{1}\right) \Omega\right) \\
=\lim _{T \rightarrow \infty}\left(\Psi^{\prime} \mid \Phi_{-}^{\text {out }}\left(F_{2}\right) \int h_{T}(t) e^{i(H-P) t} \int d \boldsymbol{x}\left(L^{*} L\right)(\boldsymbol{x}) \Phi_{+}^{\text {out }}\left(F_{1}\right) \Omega\right) \\
=\left(\Psi^{\prime} \mid \Phi_{-}^{\text {out }}\left(F_{2}\right) P_{+} \int d \boldsymbol{x}\left(L^{*} L\right)(\boldsymbol{x}) \Phi_{+}^{\text {out }}\left(F_{1}\right) \Omega\right),
\end{aligned}
$$

where in the first step we made use of the fact that $\Phi_{+}^{\text {out }}\left(F_{1}\right) \Omega=P_{+} \Phi_{+}^{\text {out }}\left(F_{1}\right) \Omega$, in the second step we exploited the invariance of $\int d \boldsymbol{x}\left(L^{*} L\right)(\boldsymbol{x})$ under translations in space and in the last step we made use of the mean ergodic theorem as in the proof of Lemma 1 of [5]. Next, we obtain

$$
\begin{aligned}
& \left(\Phi_{+}^{\text {out }}\left(F_{1}^{\prime}\right) \Phi_{-}^{\text {out }}\left(F_{2}^{\prime}\right) \Omega \mid \Phi_{-}^{\text {out }}\left(F_{2}\right) P_{+} \int d \boldsymbol{x}\left(L^{*} L\right)(\boldsymbol{x}) \Phi_{+}^{\text {out }}\left(F_{1}\right) \Omega\right) \\
& =\left(\Phi_{-}^{\text {out }}\left(F_{2}\right)^{*} \Phi_{-}^{\text {out }}\left(F_{2}^{\prime}\right) \Omega \mid \Phi_{+}^{\text {out }}\left(F_{1}^{\prime}\right)^{*} P_{+} \int d \boldsymbol{x}\left(L^{*} L\right)(\boldsymbol{x}) \Phi_{+}^{\text {out }}\left(F_{1}\right) \Omega\right) \\
& =\left(\Omega \mid \Phi_{-}^{\text {out }}\left(F_{2}^{\prime}\right)^{*} \Phi_{-}^{\text {out }}\left(F_{2}\right) \Omega\right)\left(\Omega \mid \Phi_{+}^{\text {out }}\left(F_{1}^{\prime}\right)^{*} \int d \boldsymbol{x}\left(L^{*} L\right)(\boldsymbol{x}) \Phi_{+}^{\text {out }}\left(F_{1}\right) \Omega\right),
\end{aligned}
$$

where we made use of the facts that $\left[\Phi_{+}^{\text {out }}\left(F_{1}\right), \Phi_{-}^{\text {out }}\left(F_{2}\right)\right]=0$ and that $\mathcal{H}_{+} / \mathbb{C} \Omega$ is orthogonal to $\mathcal{H}_{-} / \mathbb{C} \Omega$ (as in the proof of Lemma 4 (a) of [5]). The last term on the r.h.s. of (A.17) is treated analogously.

We note that any $\Psi_{ \pm} \in P_{E} \mathcal{H}_{ \pm}$can be approximated by a sequence of vectors of the form $P_{ \pm} F_{n} \Omega$, where $F_{n} \in \mathfrak{A}$ are quasilocal and have energy-momentum transfers in some fixed compact set. Hence, any $\Psi=\Psi_{+} \stackrel{\text { out }}{\times} \Psi_{-}$has bounded energy. By 
the above considerations and Theorem 2.9, we obtain for any $\Psi=\Psi_{+} \stackrel{\text { out }}{\times} \Psi_{-}, \Psi^{\prime}=$ $\Psi_{+}^{\prime} \stackrel{\text { out }}{\times} \Psi_{-}^{\prime}, \Psi_{ \pm}, \Psi_{ \pm}^{\prime} \in P_{E} \mathcal{H}_{ \pm}$,

$$
\begin{aligned}
\lim _{T \rightarrow \infty}\left(\Psi^{\prime} \mid Q_{T} \Psi\right)= & \left(\Psi_{+}^{\prime} \mid \Psi_{+}\right) \int d \boldsymbol{x}\left(\Psi_{-}^{\prime} \mid\left(L^{*} L\right)(\boldsymbol{x}) \Psi_{-}\right) \\
& +\left(\Psi_{-}^{\prime} \mid \Psi_{-}\right) \int d \boldsymbol{x}\left(\Psi_{+}^{\prime} \mid\left(L^{*} L\right)(\boldsymbol{x}) \Psi_{+}\right) .
\end{aligned}
$$

Now in view of Lemma A.2, any $\Psi \in P_{E} \mathcal{H}^{\text {out }}$ has the form

$$
\Psi=\sum_{m, n} c_{m, n} e_{+, m} \stackrel{\text { out }}{\times} e_{-, n}
$$

where $\left\{e_{ \pm, m}\right\}_{m=0}^{\infty}$ are orthonormal systems in $\left\{P_{E} \mathcal{H}_{ \pm}\right\}$, which we choose so that $e_{ \pm, 0}=$ $\Omega$. Defining

$$
\Psi_{+, n}=\sum_{m} c_{m, n} e_{+, m}, \quad \Psi_{-, n}=\sum_{m} c_{n, m} e_{-, m}
$$

we obtain $\rho_{ \pm, \Psi}(\cdot)=\sum_{n}\left(\Psi_{ \pm, n} \mid \cdot \Psi_{ \pm, n}\right)$. Relation (A.20) gives

$$
\lim _{T \rightarrow \infty}\left(\Psi \mid Q_{T} \Psi\right)=\int d \boldsymbol{x}\left(\rho_{+, \Psi}+\rho_{-, \Psi}\right)\left(\left(L^{*} L\right)(\boldsymbol{x})\right)
$$

Exploiting the Cauchy-Schwarz inequality and the following bounds, valid for $L=$ $A B, A \in \mathfrak{A}, B \in \mathcal{L}_{0}$,

$$
\begin{aligned}
\left|\left(\Psi \mid Q_{T} \Psi\right)\right| & \leq\left\|P_{E} \int d \boldsymbol{x}\left(B^{*} B\right)(\boldsymbol{x}) P_{E}\right\|\left\|A^{*} A\right\|, \\
\int d \boldsymbol{x}\left(\rho_{+, \Psi}+\rho_{-, \Psi}\right)\left(\left(L^{*} L\right)(\boldsymbol{x})\right) & \leq 2\left\|P_{E} \int d \boldsymbol{x}\left(B^{*} B\right)(\boldsymbol{x}) P_{E}\right\|\left\|A^{*} A\right\|,
\end{aligned}
$$

one extends (A.23) to any $L \in \mathcal{L}$. Now formula (2.36) follows by a polarization argument.

Let us now show that $\psi_{\Psi}^{\text {out }}=0$ only if $\Psi \in \mathbb{C} \Omega$. By the above considerations we obtain, for any $B \in \mathcal{L}_{0}$,

$$
\psi_{\Psi}^{\text {out }}(B, B)=\sum_{n} \int d \boldsymbol{x}\left\{\left(\Psi_{-, n} \mid\left(B^{*} B\right)(\boldsymbol{x}) \Psi_{-, n}\right)+\left(\Psi_{+, n} \mid\left(B^{*} B\right)(\boldsymbol{x}) \Psi_{+, n}\right)\right\}
$$

If $\psi_{\Psi}^{\text {out }}=0$, then $B \Psi_{ \pm, n}=0$ for each $n$ and any such $B$. Thus, by Lemma A.1 (a), $\Psi_{ \pm, n}$ are proportional to $\Omega$. Using definitions (A.21), (A.22) and the convention $e_{ \pm, 0}=\Omega$, it is easily seen that $\Psi$ is proportional to $\Omega$. 


\section{B. Proofs of Lemmas 3.7 and 3.8}

Proof of Lemma 3.7. As for the main part of the lemma, it suffices to show that the spectrum of $V_{\text {odd }}$ coincides with $\mathbb{R}_{+}$. It follows from the assumption $\operatorname{Ad} W \neq \mathrm{id}$ and the Reeh-Schlieder property of the net $(\mathcal{A}, V)$ that $\mathcal{A}_{\text {odd }}(\mathcal{I}) \neq\{0\}$ and $\mathcal{K}_{\text {odd }}=\left[\mathcal{A}_{\text {odd }}(\mathcal{I}) \Omega_{0}\right]$ for any open, bounded subset $\mathcal{I} \subset \mathbb{R}$. Let $P(\cdot)$ be the spectral measure of $V$ and suppose that $P(\Delta) \mathcal{K}_{\text {odd }}=\{0\}$ for some open subset $\Delta \subset \mathbb{R}_{+}$. We fix a non-zero $A \in \mathcal{A}_{\text {odd }}(\mathcal{I})$. Then, for any $B \in \mathcal{A}(\mathcal{I})$ the distribution

$$
\left(\Omega_{0} \mid[B, \widetilde{A}(\omega)] \Omega_{0}\right)=\frac{1}{\sqrt{2 \pi}} \int d t e^{-i \omega t}\left(\Omega_{0} \mid\left[B, \beta_{t}(A)\right] \Omega_{0}\right)
$$

is supported outside of $\Delta \cup-\Delta$. Since, by locality, this distribution is a holomorphic function, it must be zero for all $\omega \in \mathbb{R}$. Thus for any $f \in S(\mathbb{R})$ s.t. $\tilde{f}$ is supported in the interior of $\mathbb{R}_{+}$we obtain $\left(\Omega_{0} \mid B A(f) \Omega_{0}\right)=\left(\Omega_{0} \mid B \tilde{f}(T) A \Omega_{0}\right)=0$. Here $T \geq 0$ is the generator of $V, A(f)=\int d t \beta_{t}(A) f(t)$ and we made use of the fact that $A(f)^{*} \Omega_{0}=0$, due to the support property of $\tilde{f}$ and the spectrum condition. Approximating the characteristic function of the interior of $\mathbb{R}_{+}$with such $\tilde{f}$ and making use of the fact that $\left(\Omega_{0} \mid A \Omega_{0}\right)=0$, we conclude that $A \Omega_{0}=0$ and hence, by the Reeh-Schlieder property $A=0$, which contradicts our assumption. Consequently, $P(\Delta) \mathcal{K}_{\text {odd }} \neq\{0\}$ for any open subset $\Delta$ of $\mathbb{R}_{+}$, which means that the spectrum of $V_{\text {odd }}$ coincides with $\mathbb{R}_{+}$.

This fact can also be proven as follows: The representation of translations $V$ can be extended to a representation of the $a x+b$ group thanks to the Borchers theorem [27]. There is only one non-trivial, irreducible representation of this group which has positive energy [38] and its spectrum of translations is $\mathbb{R}_{+}$. Since $\mathcal{K}_{\text {odd }}$ does not contain non-trivial invariant vectors of $V$, the spectrum of $V \mid \mathcal{K}_{\text {odd }}$ coincides with $\mathbb{R}_{+}$.

Let us now proceed to part (a) of the lemma. To show the irreducibility of $\pi_{\text {odd }}$, it suffices to check that any vector $\Psi \in \mathcal{K}_{\text {odd }}$ is cyclic under the action of $\pi_{\text {odd }}\left(\mathcal{A}_{\mathrm{ev}}\right)$. By contradiction, we suppose that there is $\Psi^{\prime} \in \mathcal{K}_{\text {odd }}$ s.t. $\left(\Psi^{\prime} \mid A \Psi\right)=0$ for any $A \in \mathcal{A}_{\mathrm{ev}}$. But this implies that $\left(\Psi^{\prime} \mid B \Psi\right)=0$ for any $B \in \mathcal{A}$, which contradicts the irreducibility of the action of $\mathcal{A}$ on $\mathcal{K}$. Next we verify the faithfulness of $\pi_{\text {odd }}$ restricted to a local algebra. Let $A \in \mathcal{A}_{\mathrm{ev}}(\mathcal{I})$ be a positive local element which is zero upon restriction to $\mathcal{K}_{\text {odd }}$. For any local odd element $B \in \mathcal{A}_{\text {odd }}(\mathfrak{J})$ and for sufficiently large $s$ we obtain

$$
0=\left(\Omega \mid \beta_{s}\left(B^{*}\right) A \beta_{s}(B) \Omega\right)=\left(\Omega \mid \beta_{s}\left(B^{*} B\right) A \Omega\right) \rightarrow\left(\Omega \mid B^{*} B \Omega\right) \cdot(\Omega \mid A \Omega),
$$

where in the last step we took the limit $s \rightarrow \infty$. By the Reeh-Schlieder property it follows that $A=0$. This implies that $\pi_{\text {odd }}$ is faithful on $\mathcal{A}_{\mathrm{ev}}(\mathcal{I})$ by Proposition 2.3.3 (3) of [17]. Now the faithfulness of $\pi_{\text {odd }}$ on the quasilocal algebra $\mathcal{A}_{\mathrm{ev}}$ follows from Proposition 2.3.3 (2) of [17], which says that $\pi_{\text {odd }}$ is faithful, if and only if $\left\|\pi_{\text {odd }}(A)\right\|=\|A\|$ for any $A \in \mathcal{A}_{\mathrm{ev}}$. Local normality of $\pi_{\mathrm{odd}}$ is obvious, since $\pi_{\text {odd }}$ acts by the restriction to a subspace. Indeed, making use of Lemma 2.4.19 from [17] and of the fact that $\pi_{\text {odd }}$ preserves the norm, it is easy to check that l.u.b. $\pi_{\text {odd }}\left(A_{\alpha}\right)=\pi_{\text {odd }}$ (l.u.b. $\left.A_{\alpha}\right)$, where l.u.b denotes the least upper bound and $\left\{A_{\alpha}\right\}_{\alpha \in \mathbb{I}}$ is a uniformly bounded increasing net of positive operators from some $\mathcal{A}_{\mathrm{ev}}(\mathcal{I})$.

Part (b) of the lemma follows from the uniqueness of the invariant vector of $V$.

Proof of Lemma 3.8. We know from Lemma 3.7 that $\mathcal{A}_{\mathrm{ev}} \neq \mathbb{C} I$, since it can be irreducibly represented on the infinite dimensional Hilbert space $\mathcal{K}_{\text {odd }}$. Consequently, we can find a non-zero $A \in \mathcal{A}_{\mathrm{ev}}(\mathcal{I})$, for some open, bounded $\mathcal{I}$, s.t. $\left(\Omega_{0} \mid A \Omega_{0}\right)=0$. Proceeding identically as in the proof of the main part of Lemma 3.7, we conclude that the spectrum 
of $V_{\mathrm{ev}}$ coincides with $\mathbb{R}_{+}$. Part (b) follows trivially from the fact that the net $(\mathcal{A}, V)$ is in a vacuum representation. Irreducibility in part (a) follows from part (b). The remaining part of the statement is proven analogously as the corresponding part of Lemma 3.7.

\section{References}

1. Araki, H.: Mathematical theory of quantum fields. Oxford: Oxford University Press, 1999

2. Araki, H., Haag, R.: Collision cross sections in terms of local observables. Commun. Math. Phys. 4, 77-91 (1967)

3. Bischoff, M., Meise, D., Rehren, K.-H., Wagner, I.: Conformal quantum field theory in various dimensions. Bulg. J. Phys. 36(3), 170-185 (2009)

4. Borchers, H.-J., Buchholz, D.: The energy-momentum spectrum in local field theories with broken Lorentz-symmetry. Commun. Math. Phys. 97, 169-185 (1985)

5. Buchholz, D.: Collision theory for waves in two dimensions and a characterization of models with trivial $S$-matrix. Commun. Math. Phys. 45, 1-8 (1975)

6. Buchholz, D.: Collision theory for massless bosons. Commun. Math. Phys. 52, 147-173 (1977)

7. Buchholz, D.: The physical state space of quantum electrodynamics. Commun. Math. Phys. 85, 49-71 (1982)

8. Buchholz, D.: Gauss' law and the infraparticle problem. Phys. Lett. B 174, 331-334 (1986)

9. Buchholz, D.: Particles, infraparticles and the problem of asymptotic completeness. In: VIII ${ }^{\text {th }}$ International Congress on Mathematical Physics. Marseille 1986. Singapore: World Scientific, 1987

10. Buchholz, D.: Harmonic analysis of local operators. Commun. Math. Phys. 129, 631-641 (1990)

11. Buchholz, D.: On the manifestations of particles. In: Mathematical Physics Towards the $21^{\text {st }}$ Century. Proceedings Beer-Sheva 1993, Sen, R.N., Gersten, A., eds. Beer-Sheva: Ben-Gurion University of the Negev Press, 1994, pp.177-202

12. Buchholz, D.: Quarks, gluons, colour: facts or fiction? Nucl. Phys. B 469, 333-353 (1996)

13. Buchholz, D., Fredenhagen, K.: Locality and the structure of particle states. Commun. Math. Phys. 84, 1-54 (1982)

14. Buchholz, D., Lechner, G., Summers, S.J.: Warped convolutions, Rieffel deformations and the construction of quantum field theories. Commun. Math. Phys. 304, 95-123 (2011)

15. Buchholz, D., Mack, G., Todorov, I.: The current algebra on the circle as a germ of local field theories. Nucl. Phys. B Proc. Suppl. 5B, 20-56 (1988)

16. Buchholz, D., Porrmann, M., Stein, U.: Dirac versus Wigner. Towards a universal particle concept in local quantum field theory. Phys. Lett. B 267, 377-381 (1991)

17. Brattelli, O., Robinson, D.W.: Operator algebras and quantum statistical mechanics 1. BerlinHeidelberg-New York: Springer-Verlag, 1979

18. Chen, T., Fröhlich, J., Pizzo, A.: Infraparticle scattering states in non-relativistic QED. I. The BlochNordsieck paradigm. Commun. Math. Phys. 294, 761-825 (2010)

19. Chen, T., Fröhlich, J., Pizzo, A.: Infraparticle scattering states in nonrelativistic quantum electrodynamics. II. Mass shell properties. J. Math. Phys. 50, 012103 (2009)

20. Davidson, K.R.: $C^{*}$-Algebras by Example. Providence, RI: Amer. Math. Soc., 1996

21. Dereziński, J., Gérard, C.: Scattering theory of infrared divergent Pauli-Fierz Hamiltonians. Ann. Henri Poincaré 5, 523-577 (2004)

22. Dybalski, W.: Haag-Ruelle scattering theory in presence of massless particles. Lett. Math. Phys. 72, 27-38 (2005)

23. Dybalski, W.: Spectral theory of automorphism groups and particle structures in quantum field theory. Ph.D. Thesis, Universität Göttingen, 2008, available at http://webdoc.sub.gwdg.de/diss/2009/dybalski

24. Dybalski, W.: Continuous spectrum of automorphism groups and the infraparticle problem. Commun. Math. Phys. 300, 273-299 (2010)

25. Dybalski, W., Tanimoto, Y.: Asymptotic completeness in a class of massless relativistic quantum field theories. Commun. Math. Phys. 305, 427-440 (2011)

26. Enss, V.: Characterization of particles by means of local observables. Commun. Math. Phys. 45, 35-52 (1975)

27. Florig, M.: On Borchers' theorem. Lett. Math. Phys. 46, 289-293 (1998)

28. Fröhlich, J.: On the infrared problem in a model of scalar electrons and massless, scalar bosons. Ann. Inst. H. Poincaré Sect. A (N.S.) 19, 1-103 (1973)

29. Fröhlich, J.: Existence of dressed one electron states in a class of persistent models. Fortschr. Phys. 22, 159-198 (1974)

30. Fröhlich, J., Morchio, G., Strocchi, F.: Charged sectors and scattering states in quantum electrodynamics. Ann. Phys. 119, 241-284 (1979) 
31. Gabbiani, F., Fröhlich, J.: Operator algebras and conformal field theory. Commun. Math. Phys. 155, 569-640 (1993)

32. Haag, R.: Local quantum physics. Second edition. Berlin: Springer-Verlag, 1996

33. Hasler, D., Herbst, I.: Absence of ground states for a class of translation invariant models of non-relativistic QED. Commun. Math. Phys. 279, 769-787 (2008)

34. Herdegen, A.: Infrared problem and spatially local observables in electrodynamics. Ann. Henri Poincaré 9, 373-401 (2008)

35. Johannsen, K.: Teilchenaspekte im Schroermodell. Diplomarbeit, Universität Hamburg, 1991

36. Kawahigashi, Y., Longo, R.: Classification of two-dimensional local conformal nets with $c<1$ and 2-cohomology vanishing for tensor categories. Commun. Math. Phys. 244, 63-97 (2004)

37. Kawahigashi, Y., Longo, R.: Classification of local conformal nets. Case $c<1$. Ann. Math. 160, 493-522 (2004)

38. Longo, R.: Lectures on Conformal Nets. Real Hilbert subspaces, modular theory, $\operatorname{SL}(2, \mathbf{R})$ and CFT. In: Von Neumann algebras in Sibiu: Conference Proceedings. Bucharest: Theta, 2008, pp. 33-91

39. Pizzo, A.: One-particle (improper) states in Nelson's massless model. Ann. Henri Poincaré 4, 439-486 (2003)

40. Pizzo, A.: Scattering of an infraparticle: the one particle sector in Nelson's massless model. Ann. Henri Poincaré 5, 553-606 (2005)

41. Porrmann, M.: Particle weights and their disintegration I. Commun. Math. Phys. 248, 269-304 (2004)

42. Porrmann, M.: Particle weights and their disintegration II. Commun. Math. Phys. 248, 305-333 (2004)

43. Rehren, K.-H.: Chiral observables and modular invariants. Commun. Math. Phys. 208, 689-712 (2000)

44. Rejzner, K.: Asymptotic algebra of fields in quantum electrodynamics. Master's thesis, University of Cracow, 2009

45. Sakai, S.: $C^{*}$-algebras and $W^{*}$-algebras. Berlin-Heidelberg-New York: Springer, 1971

46. Schroer, B.: Infrateilchen in der Quantenfeldtheorie. Fortschr. Phys. 11, 1-31 (1963)

47. Stein, U.: Zur Konstruktion von Streuzuständen mit Hilfe lokaler Observabler. Ph.D. Thesis, Universität Hamburg, 1989

48. Steinmann, O.: Perturbative quantum electrodynamics and axiomatic field theory. Berlin-HeidelbergNew York: Springer, 2000

49. Takesaki, M.: Theory of operator algebras I. Berlin-Heidelberg- New York: Springer 1979

50. Wigner, E.P.: On unitary representations of the inhomogeneous Lorentz group. Ann. Math. 40, 149-204 (1939)

51. Wassermann, A.: Operator algebras and conformal field theory. III. Fusion of positive energy representations of LSU(N) using bounded operators. Invent. Math. 133(3), 467-538 (1998)

Communicated by Y. Kawahigashi 